\title{
76. Histoire externe du français au Canada, en Nouvelle-Angleterre et à Saint- Pierre et Miquelon.
}

\author{
1. Panorama historique. \\ 2. Origines, substrats et adstrats. \\ 3. Les études linguistiques. \\ 4. Le problème de la norme. \\ 5. Bibliographie sélective.
}

\section{Introduction.}

L'histoire du français en Amérique du Nord débute au XVII ${ }^{\mathrm{e}}$ s. en Acadie (l'actuelle Nouvelle-Écosse) et dans la vallée du SaintLaurent (alors appelée «Canada»); elle englobe aussi très tôt la Louisiane ( $\varnothing$ art. 77), puis l'Ontario et l'Ouest canadien, ainsi qu'un peu plus tard la Nouvelle-Angleterre. La Conquête anglaise, avec ses conséquences géo-politiques et sociales, est l'élément le plus déterminant de cette histoire mouvementée. Quant aux particularités du français des îles de Saint-Pierre et Miquelon, seul vestige territorial resté en possession de la France, elles montrent une parenté certaine avec l'acadien, qui a d'ailleurs essaimé dans les provinces maritimes et à Terre-Neuve (Port-au-Port). Seront considérés ci-dessous les facteurs géographiques, démographiques, politiques, juridiques, sociologiques, éducationnels et culturels qui caractérisent l'histoire externe des variétés de français à l'étude.

\section{Panorama historique.}

\subsection{L'Acadie.}

Port Royal (aujourd'hui Annapolis, N.-É.), le premier établissement français en Acadie (de Arcadie, dénomination due à Verrazzano), fut fondé par Champlain en 1605, mais les premières familles de colons qui devaient y faire souche ne s'y installèrent qu'à partir d'env. 1636. Lors du recensement de 1671, qui ne dénombre encore que 320 âmes, le fond de la population acadienne est déjà constitué. Plus de la moitié des quelque cinquante premiers noms de famille proviennent du centre-ouest de la France. Au moment où la colonie passe aux mains des Anglais (traité d'Utrecht, 1713), la population s'élève à env. 2500 âmes et se répartit dans quelques seigneuries. Les Acadiens sont d'abord tolérés par la Couronne anglaise, qui leur accorde le droit de rester neutres; pendant la première moitié du XVIII ${ }^{e}$ s., leur population progresse régulièrement, malgré la quasiabsence de nouveaux apports migratoires, pour atteindre env. 14000 âmes au milieu du siècle. En 1755, le refus des Acadiens de prêter serment d'allégeance à l'Angleterre entraîne leur expulsion et leur déportation, épisode tragique passé à l'Histoire sous le nom de «Grand Dérangement». Les terres sont confisquées, les établissements incendiés, et la population dispersée: des milliers de réfugiés acadiens sont déportés vers les colonies anglaises et même vers la France. Certains d'entre eux iront s'installer en Lousiane $(\varnothing$ art. 77); d'autres trouveront refuge à Saint-Pierre et Miquelon (v. cidessous 1.6.) et aux Îles-de-la-Madeleine d'où ils iront essaimer à Terre-Neuve (v. cidessous 1.7.) et sur la Basse-Côte-Nord-, mais plusieurs d'entre eux reviendront s'établir, à la fin de la guerre de Sept Ans, en Nouvelle-Ecosse et à l'île du PrinceÉdouard, où ils formeront des isolats qui ont subsisté jusqu'à nos jours. C'est toutefois au Nouveau-Brunswick que le plus grand nombre d'entre eux est aujourd'hui regroupé; s'y ajoutent deux régions contiguës, le Madawaska américain (dans le nord du Maine) et le sud de la Gaspésie (région de Carleton, sur la Baie des Chaleurs). Enfin, plusieurs viendront s'installer dans la vallée laurentienne, y fondant çà et là de nouveaux villages qu'on appellera «Petites Cadies»; ils semble qu'ils se soient intégrés toutefois assez tôt à l'ensemble québécois.

Le taux de natalité extrêmement élevé des Acadiens (comparable à celui des Québécois) leur permet de survivre en tant que peuple: alors que seulement 2000 réfugiés s'y étaient installés après la déportation, le Nouveau-Brunswick comptait un siècle plus tard près de cinquante mille descendants acadiens; en 1941, malgré l'immigration massive vers la Nouvelle-Angleterre (v. ci- 
dessous 1.5.), ce chiffre avait plus que triplé (Massignon 1962:79), et selon le dernier recensement le nombre de locuteurs ayant le français comme langue maternelle dans cette province s'élève à quasi 240000 personnes (33\% d'une population totale d'env. 730000 âmes, v. tableau 1b); le nombre de ceux qui ont le français comme langue d'usage à la maison y est un peu plus bas (env. 220000 , v. tableau 2b), mais le nombre total de francophones, en tenant compte des bilingues, atteint plus de 310000 locuteurs (42\%, v. tableau 3; sur l'anglicisation au Nouveau-Brunswick, v. Castonguay 1996). En Nouvelle-Écosse, les personnes ayant le français comme langue maternelle ne représentent que $3,9 \%$ de la population ( $t$. 1b); le taux d'assimilation y est élevé, avec seulement $2,2 \%$ ayant le français comme langue d'usage à la maison (t. 2b), mais en tenant compte des bilingues la connaissance du français s'élève à 9,5\% (t. 3). La situation est très semblable à l'île-du-Prince-Édouard, avec respectivement $4,2 \%$ (langue maternelle), $2,2 \%$ (à la maison), et $11,1 \%$ (bilingues).

De la Conquête anglaise à l'Acte de 1'Amérique du Nord britannique (1867, v. cidessous 1.2.3.), la présence des Acadiens dans les provinces maritimes est «tolérée»; le clergé catholique, pilier de la survivance culturelle et linguistique, réussit à y maintenir un enseignement primaire en français. $\mathrm{La}$ Constitution de la Confédération canadienne naissante protège vaguement les droits des minorités linguistiques dans le domaine de l'enseignement, tout en reconnaissant que ceux-ci relèvent de la juridiction provinciale; les gouvernements provinciaux étant dominés par l'élément anglais, le système scolaire francophone dans les Maritimes se trouve privé d'un soutien actif de l'État et ne survit que grâce à la volonté populaire: «La perception des taxes destinées à alimenter les écoles du Nouveau-Brunswick provoqua une échauffourée à Caraquet (Gloucester) en 1875 , et ce fut le signal d'un réveil chez les Acadiens, décidés à procurer à leurs enfants un enseignement tenant compte de leur langue et de leur foi. Soutenus par un clergé actif et entreprenant, et des congrégations venues de France ou de la Province de Québec, ou recrutées sur place, ils mirent sur pied, entièrement à leurs frais, des écoles primaires et même des collèges secondaires.» (Massignon 1962:82). Depuis 1969, le Nouveau-Brunswick est officiellement bilingue; depuis 1981, une nouvelle loi linguistique reconnaît l'égalité des deux communautés linguistiques officielles de cette province (Péronnet 1995:403). Les francophones $y$ possèdent en outre des périodiques (dont un quotidien, L'Acadie nouvelle, publié à Caraquet, quelques hebdomadaires et un mensuel), de nombreuses stations de radio et une station de télévision en langue française (Radio-Canada à Moncton), ainsi qu'un établissement d'enseignement supérieur (l'Université de Moncton). Quant à la Nouvelle-Écosse et à l'̂̂le-du-PrinceÉdouard, le français n'y jouit d'aucun statut particulier (sauf pour ce qui relève de l'État fédéral) et l'assimilation y est très forte. On notera toutefois l'existence à la Baie SainteMarie (Nouvelle-Écosse) d'un établissement d'enseignement supérieur, l'Université Sainte-Anne; la province connaît aussi un hebdomadaire francophone, Le Courrier de la Nouvelle-Écosse (Yarmouth), dont le pendant à l'île-du-Prince-Édouard s'appelle La Voie acadienne (Summerside). Une radio régionale en français (CBAF) a des bureaux à Halifax (N.-É.) et Charlottetown (Î.-P.-É.).

Dans le domaine littéraire, on rappellera le nom d'Antonine Maillet (née à Bouctouche [N.-B.] en 1929), prix Goncourt 1979 pour Pélagie-la-Charrette et porte-drapeau de l'identité acadienne.

\subsection{Le «Canada» (la colonie lauren- tienne).}

La vallée du Saint-Laurent («Canada» sous le régime français, «Province de Québec», «Bas-Canada» puis «Canada-Est» pendant le régime anglais, et à nouveau «Province de Québec» depuis 1867) est le berceau de l'autre plus ancienne communauté francophone d'Amérique. La langue française, au prix de luttes perpétuelles, a réussi à 
s'y maintenir et à y prospérer, dans un premier temps grâce à l'isolement, à la cohésion et à l'homogénéité religieuse et culturelle des locuteurs - le tout conjugué à un taux de natalité exceptionnellement élevé -, puis par des moyens politiques et législatifs.

\subsubsection{Le régime français.}

Champlain fonde Québec en 1608, mais ce n'est qu'à partir d'env. 1633 que la Compagnie des Cent-Associés entreprend d'installer des colons, principalement à Québec, Trois-Rivières (fondé en 1634) et Montréal (fondé en 1642). Peu à peu, les deux rives du Saint-Laurent sont défrichées et cultivées, et la colonisation avance vers l'intérieur des terres. Pendant les premières décennies, l'apport migratoire est régulier mais reste faible: env. mille quatre cents pionniers étaient venus s'établir au «Canada» en 1663, date à laquelle le gouvernement royal décide de favoriser la colonisation en permettant l'établissement des soldats et en organisant le départ de jeunes femmes célibataires (les fameuses «Filles du Roy», des orphelines parisiennes) pour corriger la prépondance démographique de l'élément masculin dans la colonie. Durant la période 16631679, 1983 pionniers s'installent au pays (Charbonneau / Guillemette 1994:61), venant former avec leurs prédécesseurs la base de la population d'origine française de la vallée laurentienne; en effet, le nombre de personnes venues en Nouvelle-France pour s'y installer diminue vers la fin du XVII ${ }^{\mathrm{e}} \mathrm{s}$. et reste assez faible jusqu'à la Conquête, les nouveaux arrivants se fondant dans la masse des habitants nés au pays. Le nombre total d'individus ayant fait la traversée pendant l'ensemble de la période coloniale est évalué à env. trente mille, mais moins du tiers ont fait souche (Bouchard / Tremblay 1995:310311); la formidable expansion démographique de la colonie au XVIII ${ }^{\text {e }}$ s. est essentiellement due à l'accroissement naturel de la population. On évalue le nombre d'habitants à env. 3200 en 1666, puis à près de 10000 en 1681, et enfin à quelque 70000 à la Conquête (1760); v. Bouchard / Tremblay 1995:321.

Les origines régionales des colons de la vallée du Saint-Laurent pour la période fondatrice de 1608-1679 sont caractérisées par l'importance des contingents normandspercherons $(22,9 \%)$, franciliens $(17,8 \%)$ et du Centre-Ouest (28\%); v. Charbonneau / Guillemette 1994:66. On rappellera que la proportion de pionniers originaires de cette dernière région était beaucoup plus importante en Acadie (env. 50\%, v. ci-dessus 1.1.), qui ne connut que très peu de colons normands et parisiens (v. Massignon 1962:74). Si l'on distingue la période 16081662 de la période 1663-1679, les faits les plus marquants sont l'importance de la région Normandie-Perche dans la première tranche $(30,4 \%)$, et la supériorité numérique de l'élément féminin originaire de la région parisienne dans la seconde (30,3\%); v. Charbonneau / Guillemette 1994:66. Les colons sont en moyenne plus urbains et plus instruits que la moyenne de leurs compatriotes: six pionniers sur dix déclarent une ville ou un bourg comme lieu d'origine (id. 68). On notera en outre que la présence d'une classe dirigeante francophone et le contact avec la norme métropolitaine se sont maintenus plus longtemps dans la colonie laurentienne qu'en Acadie, dont le français a conservé un plus grand nombre de traits archaïsants, populaires ou dialectaux. Avec la Conquête, toutefois, une partie des élites «canadiennes» retourne en France et le peuple est laissé à lui-même; sa langue continue à vivre et à se développer, indépendamment des évolutions métropolitaines mais bientôt influencée par le contact avec la langue des nouveaux occupants, l'anglais.

\subsubsection{Le régime anglais.}

En septembre 1759, Québec tombe aux mains des Anglais; un an plus tard, Montréal capitule à son tour. Un régime militaire est instauré, qui sera maintenu jusqu'à la fin de la Guerre de Sept Ans, scellée par le Traité de Paris (1763); la France renonce définitivement à ses colonies 
du nord-est de l'Amérique, qui se retrouvent sous tutelle britannique. Contrairement à ce qui s'est passé en Acadie, la population n'est pas violentée ni expulsée par l'armée anglaise, qui a reçu comme directive de vivre en bonne intelligence avec le peuple. $\mathrm{La}$ supériorité numérique des soixante-dix mille «Canadiens» de la vallée laurentienne rendait en fait cette clémence nécessaire. Le statut juridique de la langue française dans les structures politiques et législatives allait toutefois connaître de nombreux avatars tout au long du régime anglais.

\subsubsection{La proclamation royale (1764).}

Pendant le régime militaire (17591764), les lois et coutumes françaises ne furent pas abolies en matière civile; les gouverneurs anglais connaissaient le français, ou se faisaient assister par des secrétaires suisses ou huguenots, et les affaires civiles continuèrent d'être administrées par des «Canadiens» (Noël 1990:75). À la suite du Traité de Paris, la «Proclamation royale», effective à partir de 1764 , est le premier texte concernant l'organisation de la nouvelle colonie. La langue n'y est pas explicitement évoquée; on y stipule toutefois que l'administration de la justice et la formation d'une assemblée représentative devra se faire dans le respect des lois britanniques. C'est alors la religion catholique et non la langue française qui pose problème: en théorie, les «papistes» n'ont pas le droit de faire partie des cours de justice. Les premiers gouverneurs, Murray et Carleton, durent toutefois faire preuve de pragmatisme: les francophones, qui constituaient alors 99,7\% de la population (Noël 1990:65), furent autorisés à sièger comme jurés et à pratiquer comme avocats. Cette situation fut toutefois violemment critiquée par les marchands anglais: «les protestants voulaient à tout prix éviter les désavantages d'un jury unilingue francophone dans une cause entre anglophones» (Noël 1990:82). L'administration de la justice fut assez cahotique pendant toute cette période. Quant à la formation d'une assemblée représen- tative, elle fut l'objet de nombreux débats, qui se perpétuèrent dans la période suivante. Le premier journal de la colonie, La Gazette de Québec, est fondé en 1764; toutes les ordonnances y sont publiées en anglais et traduites en français. Comme les premiers colons de langue anglaise s'installent plutôt en Nouvelle-Écosse, sur les terres ayant jadis appartenu aux Acadiens (v. ci-dessus), la proportion d'anglophones dans la vallée laurentienne ne dépasse pas 3\% durant cette période.

\subsubsection{L'Acte de Québec (1774).}

L'Acte de Québec ne comporte pas de dispositions précises sur la langue, mais dans l'ensemble on peut juger qu'il est favorable aux francophones, puisque les lois françaises sont restituées au civil, que les garanties religieuses sont explicitement renouvelées, et qu'il est permis aux Catholiques d'accéder, en théorie, à toutes les fonctions administratives. Une sorte de bilinguisme s'instaure: «les avocats plaidaient dans les deux langues et les juges pouvaient, par exemple, commencer leur jugement en anglais, dire deux phrases en français, pour poursuivre en anglais [...]; les registres étaient, dans la mesure du possible, tenus dans les deux langues» (Noël 1990:113). La période 1774-1791 ne verra pas encore la formation d'une assemblée représentative; la colonie est administrée par un Conseil législatif, composé de hauts fonctionnaires britanniques mais aussi, pour un tiers, de seigneurs catholiques sans pouvoir politique réel (il s'agit de propriétaires terriens qui avaient encore à cette époque le privilège de percevoir la dîme). Les débats sont menés, consignés et publiés dans les deux langues. De nouveaux journaux voient le jour, dans lesquels on assiste à l'émergence d'un discours méta-linguistique. La connaissance de l'anglais commence déjà à s'affirmer comme instrument de promotion sociale, en particulier chez les jeunes citadins appelés à travailler comme interprètes, traducteurs ou avocats, mais la plus grande partie du peuple, encore essentiellement 
constituée à cette époque par la paysannerie, reste entièrement illettrée et n'a guère l'occasion de côtoyer les anglophones. La population, estimée à env. 90000 personnes vers 1766, atteint en 1790 les 150000 âmes (Noël 1990:69); la proportion de l'élément anglais monte à 15\%, par l'effet conjugué de l'immigration britannique et de la fuite des Loyalistes américains, dont plusieurs milliers viennent s'installer dans la colonie, entre autres dans la région des Cantons de l'Est, au sud-est de Montréal, mais surtout dans ce qui deviendrait le «Haut-Canada» (l'actuelle province de l'Ontario).

\subsubsection{L'Acte constitutionnel (1791).}

La plupart des nouveaux colons britanniques s'étant installés au sud-ouest de la vallée laurentienne, dans le territoire qu'on nomme aujourd'hui la péninsule ontarienne, le besoin de séparer la colonie - difficilement gouvernable - en deux entités politiques distinctes se fit sentir. Le «Canada» fut alors scindé en «Bas-Canada» (la vallée du SaintLaurent, très majoritairement francophone) et «Haut-Canada» (le sud de l'actuel Ontario, anglophone). Le Bas-Canada put conserver ses lois civiles françaises et sa liberté religieuse; il fut doté pour la première fois d'une Chambre d'assemblée élue, dont les pouvoirs étaient toutefois limités par l'existence d'un Conseil législatif et exécutif dont les membres étaient nommés par le gouverneur (britannique). Comme l'Acte constitutionnel ne comportait aucune disposition explicite sur le statut des langues, toute la période est caractérisée par des luttes incessantes entre francophones et anglophones sur la place qu'il convient d'accorder au français dans les structures politiques et juridiques du pays. À l'Assemblée, qui compte toujours une majorité de députés francophones, les deux langues sont en usage dans les débats, mais seul l'anglais a valeur de langue officielle pour la rédaction des lois (qui sont toutefois aussi traduites en français). Dans le domaine judiciaire, l'anglais domine: «Tous les juges en chef de la province ainsi que près des trois quarts de la magistrature étaient d'origine britannique. Dans les cours de juridiction criminelle, seul le droit anglais était employé et l'anglais était alors la seule langue utilisée.» (Noël 1990:241). Un riche discours métalinguistique, souvent passionné, embrase les pages des journaux de l'époque, les «Canadiens» réclamant le droit d'être membres de l'Empire britannique tout en conservant leur langue, les «Anglais» s'y opposant souvent avec véhémence. C'est aussi l'époque où les anglicismes commencent à apparaître en grand nombre, en particulier dans la langue parlementaire et juridique, secteurs dans lesquels le français fut très tôt restreint au statut de langue de traduction. Des tentatives d'organiser un système d'enseignement généralisé dans tout le pays voient le jour, mais se heurtent à de nombreuses difficultés (v. Noël 1990:140145). Sur le plan social, on notera la graduelle perte d'influence des seigneurs et l'émergence d'une nouvelle élite francophone issue de la petite bourgeoisie, composée de médecins, de notaires et d'avocats qui souvent représentent le peuple au Parlement, et en est très proche: "Ainsi les personnes les plus instruites de chaque village appartiennent dans la société aux mêmes familles et au même rang de naissance que les habitants illettrés [...] La plus parfaite égalité règne toujours dans leurs relations; celui qui est supérieur par l'éducation n'est séparé du paysan singulièrement ignare qui le coudoie, par aucune barrière d'usages ou de fierté ou d'intérêts.» (Lord Durham, cité dans Noël 1990:244). Cette situation a pour corollaire, sur le plan linguistique, l'absence de stratification des usages linguistiques dans la société de l'époque.

La population du Bas-Canada augmente à un rythme soutenu, nourri par la grande fécondité des «Canadiens» et par l'immigration britannique; vers 1825 , la population est de 480000 habitants mais le pourcentage de francophones est tombé à 77\% (Bouchard / Tremblay 1995:321). Les anglophones se concentrent dans les villes (à l'exception des Loyalistes des Cantons de l'est) et les francophones étendent leur 
occupation du territoire à l'Outaouais (depuis la région montréalaise), à Charlevoix (depuis Québec) et à la Gaspésie (depuis le BasSaint-Laurent).

\subsubsection{L'Acte d'Union (1840).}

À la suite des nombreux troubles ayant éclaté au sein de la colonie (en particulier la révolte des Patriotes, 1837-38), Londres décide d'entreprendre une réforme en profondeur des structures politiques en réunissant en un seul ensemble le Haut et le Bas-Canada. L'Acte d'Union, adopté en 1840 , a pour but avoué l'assimilation des francophones, et pour conséquence indirecte la diminution de leur pouvoir politique: l'article 41 stipule que l'anglais est la seule langue officielle de la législature, et la fusion des deux «Canada» en un seul système parlementaire, dans lequel le Haut-Canada anglophone occupe une place égale malgré son infériorité numérique, met les francophones du Bas-Canada en situation de minorité. L'article 41, jugé comme inacceptable par les Canadiens français, sera révoqué en 1848 à la suite de nombreuses protestations, mais il a contribué à mettre la langue française au cœur de leurs revendications politiques. Dans le domaine judiciaire, les deux langues sont admises au civil mais au criminel seul l'anglais a droit de cité. La période 1840-1867 est caractérisée par une réorganisation du système d'enseignement, les Canadiens français réussissant enfin à mettre sur pied un réseau d'écoles capables de desservir une grande partie de la population; en 1852, l'Université Laval est fondée à Québec. Alors que la période précédente avait connu quelques tentatives d'établissement d'un enseignement laïque, tout le système scolaire francophone est désormais dans les mains du clergé catholique, qui fait même venir des congrégations religieuses de France pour le seconder dans sa tâche. Ce seront les seuls immigrants français à venir au Canada au XIX ${ }^{\mathrm{e}}$ siècle, mais ils ont pu jouer un certain rôle au sein du clergé dans l'instauration et la transmission d'une norme de français «cultivé». Sur le plan social, l'anglomanie s'installe dans les classes francophones urbaines proches du pouvoir, selon le schéma classique de l'appropriation de la langue des dominants par les élites du groupe social dominé en quête d'ascension sociale. Paradoxalement, la culture d'expression française connaît à la même époque une grande vitalité, qui s'exprime par l'apparition de sociétés culturelles, la fondation d'instituts et de bibliothèques, et la publication de revues et d'ouvrages littéraires (v. DOLQ, t. 1); un discours puriste, qui fait la guerre aux anglicismes et aux barbarismes, commence à voir le jour. Sur le plan démographique, la période marque le début de l'émigration des Canadiens vers les États-Unis (v. ci-dessous 1.5.), causée par l'état de surpopulation dans lequel se trouvent les campagnes, dont toutes les terres arables sont désormais occupées: de 1840 à 1860, «un peu plus de 100000 personnes (dont les trois quarts d'origine française) auraient quitté le Québec pour se diriger vers les États de la NouvelleAngleterre» (Bouchard / Tremblay 1995:323). La population du Canada-Est atteint tout de même 1112000 habitants en 1861, dont 930000 Canadiens français (ibid. 321).

\subsubsection{L'Acte de l'Amérique du Nord britannique (1867).}

En 1867, le Nouveau-Brunswick et la Nouvelle-Écosse se joignent au Québec et à l'Ontario pour former la Confédération canadienne, qui comptait donc à ses débuts quatre provinces. Dans l'Acte de l'Amérique du Nord britannique, qui posa les bases du nouvel état fédéral, seul l'article 133 faisait mention de l'usage des langues; l'anglais et le français y sont explicitement mis sur le même pied dans les structures législatives et judiciaires du Canada (niveau fédéral) et du Québec (niveau provincial). Les autres sphères de l'activité humaine (enseignement, commerce, relations de travail, santé, affaires sociales, etc.) ne sont pas mentionnées. $\mathrm{Si}$ l'enseignement en français à tous les niveaux est assuré par le clergé catholique, qui 
s'occupe aussi des hôpitaux et des œuvres à caractère social, le commerce et le monde du travail sont dans les mains des anglophones, ce qui entraîne une forte anglicisation des champs sémantiques concernés. L'exploitation forestière et le secteur des pâtes et papier comptent parmi les principales activités économiques au tournant du siècle, et laisseront dans la langue un contingent de mots anglais; elles seront bientôt suivies par l'exploitation minière, dont l'essor entraînera après la Grande Guerre la formation d'une nouvelle zone de colonisation, l'Abitibi, linguistiquement composite. Les élites urbaines canadiennesfrançaises sont bilingues, l'anglais étant la condition sine qua non de l'avancement social; les anglophones du Québec restent quant à eux essentiellement unilingues. $\mathrm{La}$ paysannerie et le prolétariat urbain (la population du Québec, de majoritairement rurale qu'elle était au $\mathrm{XIX}^{\mathrm{e}}$ siècle, bascule et devient à plus de $50 \%$ citadine au début du $\mathrm{XX}^{\mathrm{e}}$; v. Bouchard / Tremblay 1995:320) n'échappent plus au contact avec l'anglais: d'une part, toutes les nouvelles technologies (voitures, équipement aratoire, machinerie industrielle, nouveaux produits de consommation) sont d'origine américaine; d'autre part, un important mouvement de va-et-vient entre le Québec et ses colonies linguistiques de Nouvelle-Angleterre favorise l'implantation de mots anglais jusque dans les régions les plus reculées de la province (Poirier 1995:770). Dans le domaine littéraire, la période est dominée par le roman $\mathrm{du}$ terroir (Un homme et son péché, Cl.-H. Grignon, 1933; Menaud, maître-draveur, F.A. Savard, 1937); les thématiques urbaines s'imposeront peu à peu après la Seconde Guerre Mondiale (Bonheur d'occasion, G. Roy, prix Femina 1945; Les Plouffe, R. Lemelin, 1948). Les canadianismes apparaissent timidement, d'abord en italiques ou entre guillemets, dans les passages dialogués, puis s'affranchissent peu à peu des artifices typographiques et investissent les passages narratifs. Les chroniques de langue (v. Prévost 1996) enjoignent la population à éviter les barbarismes et surtout les anglicismes; toutefois, si de nombreux anglicismes lexématiques courants au XIX ${ }^{\mathrm{e}}$ siècle sont effectivement tombés en désuétude depuis, les calques en revanche sont souvent passés inaperçus et ont échappé à la pression puriste (v. Poirier 1995:788). La période assiste en outre à la publication de l'excellent Glossaire du parler français au Canada (1930), ouvrage descriptif et différentiel réalisé par la Société du parler français au Canada. La radio fait son apparition et joue un certain rôle du point de vue linguistique, d'une part en diffusant la chanson française dans toutes les couches d'une population qui avait perdu depuis longtemps tout contact avec le français parlé en France (favorisant ainsi un début de connaissance passive de la norme européenne contemporaine), d'autre part en donnant une tribune aux journalistes, chanteurs, comédiens et humoristes québécois en qui la population se reconnaît et dont les productions langagières contribueront à former et à répandre par la voie des ondes une norme implicite pan-québécoise.

Durant cette période, la population du Québec a quintuplé, passant en un siècle d'env. un million à env. cinq millions d'habitants (Bouchard / Tremblay 1995:324), pour une proportion de francophones qui a toujours oscillé autour de $80 \%$ (30\% pour l'ensemble du Canada). Au début du $\mathrm{XX}^{\mathrm{e}}$ siècle, le phénomène de l'immigration internationale fait son apparition. Les nouveaux arrivants (d'abord Italiens, Juifs, Grecs, Allemands, Polonais, Ukrainiens, Chinois), concentrés à Montréal, rejoindront massivement le groupe des anglophones, pour des raisons socio-économiques (pouvoir d'attraction de l'anglais comme langue du commerce et de l'ascension sociale) mais aussi religieuses (le système scolaire francophone est sous la domination totale du clergé catholique, qui ne ménage aucune place dans ses structures aux membres des autres confessions).

\subsubsection{De la Révolution tranquille (1960- 1966) à l'aube du XXI' siècle.}


Après une longue période caractérisée par l'immobilisme des structures sociales, dominées par le clergé catholique et le gouvernement réactionnaire de $M$. Duplessis (époque baptisée la Grande Noirceur), la première moitié des années soixante assiste à un processus de réformes politiques, économiques et sociales menées par le nouveau gouvernement libéral de J. Lesage et qui permettent au Québec de faire son entrée dans la modernité. Cette période est passée à l'Histoire sous le nom de Révolution tranquille. L'accès à l'enseignement supérieur est démocratisé; le monde de l'éducation, de la santé et des œuvres sociales est irréversiblement sécularisé. Le boom industriel de l'après-guerre et la reprise de l'immigration rendent la société québécoise de plus en plus urbaine et multi-culturelle; en même temps, les sentiments autonomistes ou séparatistes commencent à se manifester de façon organisée. Une série de lois linguistiques (loi 63, 1969; loi 22, 1974; loi 101, 1977; v. Wolf 1987 pour leur reproduction intégrale en fac-similé) viendront préciser le statut de la langue française au Québec, faisant de celle-ci la seule langue officielle de l'État provincial, tout en reconnaissant explicitement l'existence des communautés anglophones, allophones et autochtones. La natalité des francophones ayant chuté de façon spectaculaire, la question de l'intégration des immigrants à la majorité de langue française devient cruciale. Le Québec obtient le droit de mener sa propre politique en matière d'immigration, jusque-là de compétence fédérale; il tente avec un succès limité de favoriser l'immigration en provenance de pays francophones (Haïti, Liban, Vietnam, Maghreb, France) et oblige les enfants d'immigrés à être scolarisés en français, quelle que soit leur langue d'origine. Le pouvoir d'attraction de l'anglais étant toujours aussi fort, on rencontre désormais à Montréal de nombreux trilingues; les élites francophones sont quant à elles toujours bilingues, mais la nouveauté est constituée par l'accession au bilinguisme des membres de la communauté anglophone (ceux qui ont choisi de rester au Québec), qui jusque-là n'avaient guère montré d'intérêt pour l'apprentissage du français. Dans le reste de la province, l'unilinguisme français n'est pas rare. En ce qui concerne la langue des relations de travail, du commerce et de l'affichage, la province est francisée en profondeur; un énorme effort est accompli dans le domaine de la terminologie. L'accès à la télévision, qui se démocratise dans les années cinquante et soixante, entraîne les mêmes conséquences que l'avènement de la radio: diffusion de la norme européenne à travers les films français et les films américains doublés en France (vélo remplace bicyclette, papi talonne grand-papa, et même week-end commence à se substituer à fin de semaine, etc.), et consolidation d'une norme implicite pan-québécoise connaissant divers niveaux de langue selon les types de discours. On peut citer comme exemple le vocabulaire du hockey sur glace qui, jusque là profondément anglicisé, est entièrement francisé et diffusé par les commentateurs sportifs. Les Québécois francophones ont aussi accès à des chaînes anglophones, mais la plupart d'entre eux ne les regardent guère, à moins d'avoir une excellente connaissance de l'anglais. Dans le domaine littéraire, l'utilisation consciente des niveaux de langue les plus stigmatisés, illustrée de façon spectaculaire par Michel Tremblay avec ses célèbres Belles-Sœurs (1968), répond à des fins stylistiques (recherche de l'authenticité, renouvellement de la langue littéraire à partir des ressources de l'oralité) mais aussi politiques (dénonciation des inégalités sociales).

Les données du dernier recensement (1996) révèlent que le français est la langue maternelle de $81 \%$ des sept millions de Québécois, et la langue d'usage à la maison de $82 \%$ d'entre eux; pour l'anglais, ces pourcentages sont respectivement de $8,3 \%$ et de $10,1 \%$ (v. tableaux 1 b et $2 b$ ). $38 \%$ des Québécois se déclarent bilingues anglais-français (v. tableau 3), le plus haut pourcentage au pays. Si on leur additionne les unilingues français, le pourcentage de connaissance de la langue française atteint $94 \%$ au Québec. 


\subsection{L’Ontario.}

L'histoire du français en Ontario remonte au XVII ${ }^{\mathrm{e}}$ siècle, alors que le territoire correspondant à ce qui allait devenir le HautCanada pendant le régime anglais n'est encore qu'une petite partie de l'immense territoire qui s'appellait alors la NouvelleFrance. À partir de la colonie laurentienne, des explorateurs partent à la découverte de la région des Grands Lacs, bientôt suivis par des «engagés» et des «coureurs de bois» qui assureront le commerce des fourrures en servant d'intermédiaires entre Européens et Amérindiens. Le poste de traite de la région du Détroit (correspondant approximativement aux actuelles villes de Détroit [É.-U.] et de Windsor [Ont.]) comptait, en plus des Hurons, quelques centaines d'habitants d'origine européenne dans la première moitié du XVIII siècle; c'est là qu'un jésuite belge, le père Pierre Philippe Potier, rédige le premier témoignage métalinguistique sur le français des «Canadiens», qui comportait déjà nombre de traits particuliers (1743 à 1758, v. Halford 1994). Avec la Conquête (v. ci-dessus 1.2.3.), toute immigration française est interrompue, et peu à peu la région dénommée à l'époque «les pays d'en haut» est peuplée de colons britanniques et de loyalistes américains qui réduisent dans un premier temps la part de l'élément français à un pourcentage négligeable (sauf dans la région du Détroit). Toutefois, des courants migratoires en provenance du Québec fourniront à l'Ontario des contingents de population francophone qui créeront çà et là des communautés relativement importantes, en particulier dans l'Outaouais et l'Abitibi ontariens (resp. Ottawa, Sudbury); la ville de Toronto attire aussi de nombreux Québécois, qui ont formé une diaspora francophone d'env. cinquante mille locuteurs dans la métropole du Canada anglais (plus de quatre millions d'habitants au dernier recensement). L'enseignement en français est théoriquement assuré «là où le nombre le justifie»; l'application de ce principe a toutefois souvent donné lieu à d'épiques batailles juridiques, la majorité anglophone n'étant pas toujours disposée à consentir les efforts financiers nécessaires pour que ce principe soit respecté. Dans l'enseignement supérieur, on relève l'existence de deux universités bilingues (l'Université d'Ottawa et l'Université Laurentienne à Sudbury, à laquelle est rattaché un petit collège universitaire entièrement francophone appelé Université de Hearst), ainsi que de nombreux départements de French Studies dans les universités anglophones. Le quotidien Le Droit d'Ottawa est le principal organe de presse francophone en Ontario (www.ledroit.com/); il fut fondé en 1913, en réaction au Règlement 17, qui interdisait alors l'usage du français comme langue d'enseignement dans toute la province.

Le français des Franco-Ontariens (aussi appelés Ontarois) se distingue essentiellement du français québécois par une plus grande anglicisation, phénomène bien étudié par de nombreux chercheurs, en particulier sous les angles de l'alternance codique, des phénomènes d'interférences et des problèmes d'identité ethno-linguistique (v. entre autres Chaudenson / Mougeon / Beniak 1993, Mougeon 1993 et Heller 1994); presque tous les francophones de l'Ontario sont en fait bilingues, et le taux d'assimilation y est très élevé: $4,5 \%$ des Ontariens ont le français comme langue maternelle, mais seulement $2,7 \%$ pratiquent cette langue à la maison (v. tableaux $1 b$ et $2 b$ ). Si l'on additionne les bilingues déclarés (qui comptent un bon nombre d'anglophones ayant acquis le français comme langue seconde, en particulier dans des programmes d'immersion ayant connu un certain succès) et les francophones exclusifs (v. tableau 3), on arrive à un pourcentage total de $12 \%$ d'Ontariens connaissant le français; en valeurs absolues, ces quelque 1280000 locuteurs forment le plus fort contingent à l'extérieur du Québec, dépassant de loin celui du NouveauBrunswick.

\subsection{L'ouest canadien.}

Il importe de distinguer ici deux groupes ethniques: d'une part les Canadiens fran- 
çais (Franco-Manitobains, Fransaskois, Franco-Albertains et Franco-Colombiens) descendants de colons originaires du Québec venus s'installer dans les provinces de l'Ouest depuis la première moitié du XVIIIe siècle (et jusqu'à nos jours), d'autre part les Métis, descendants des «engagés» ou «coureurs de bois» chargés de la traite des fourrures (il s'agissait de Québécois et aussi, plus tard, d'Ecossais) unis à des Amérindiennes. Le français n'est plus tellement parlé par les Métis (cf. cependant Papen 1998), mais leurs ancêtres ont laissé derrière eux un important contingent de toponymes d'origine française dans l'ouest de l'Amérique du Nord (v. Lapierre 1994); l'héritage français survit également dans les anthroponymes, ainsi que dans certaines manifestations culturelles (contes et chants populaires, musique, danse). Sur le «mitchif» de l'Ouest américain, $\varnothing$ art. 77. Le contact entre le français et les langues amérindiennes a en outre donné naissance à un jargon, le «tchinouk», qui a conservé de nombreux éléments lexicaux d'origine française (v. Lang 1995).

Quant au français des francophones de l'Ouest, il est très marqué par le contact avec l'anglais (interférences, alternance de code, taux d'assimilation élevé) mais à la base il s'agit d'une extension géographique $\mathrm{du}$ français laurentien. Largement minoritaire, il aurait pu connaître un sort meilleur si des mesures législatives n'avaient pas été prises contre lui dans les premières années de la Confédération. En 1869, le gouvernement fédéral canadien fait l'acquisition des Territoires du Nord-Ouest, alors propriété de la Compagnie de la Baie d'Hudson. En 1870, une nouvelle province est créée dans la région de la Rivière Rouge, le Manitoba; si au début le français et l'anglais y sont mis sur le même pied (art. 23 de l'Acte du Manitoba), le français est «aboli progressivement comme langue officielle [...] par une série de mesures qui s'échelonnèrent du 4 février 1889 au 28 mars 1890» (Rapport de la commission d'enquête 1972:283). L'exode d'une partie des Métis vers l'Ouest (canadien et américain), conjugué à l'arrivée de colons ontariens (anglophones), consacrera définitivement et irréversiblement le statut minoritaire de la langue française au Manitoba. Les nombreux immigrants d'Europe centrale et d'Europe de l'Est qui iront peupler les prairies canadiennes dans la première moitié du $\mathrm{XX}^{\mathrm{e}}$ siècle n'auront bien sûr d'autre choix que de scolariser leurs enfants en anglais tout comme les immigrants francophones en provenance de l'est du pays. Ce n'est qu'en 1970 que le français redevient langue d'enseignement officielle au Manitoba; en 1987, les mesures répressives dont le français avait été victime ont même été déclarées anticonstitutionnelles, entraînant l'obligation de traduire dans cette langue toutes les lois votées par le parlement provincial depuis 1890. Cette reconnaissance tardive et de nature législative et juridique ne peut toutefois corriger le déficit démographique accumulé. $\mathrm{Au}$ dernier recensement fédéral (1996), 4,3\% des Manitobains ont déclaré le français comme langue maternelle (v. tableau 1b), mais seulement $2,0 \%$ le parlent encore à la maison (v. tableau 2b); pourtant, $9,4 \%$ se déclarent bilingues (v. tableau 3), situation qu'il faut peut-être attribuer au succès des programmes d'immersion. Dans le domaine littéraire, on rappellera que la romancière Gabrielle Roy (1909-1983) est née à Saint-Boniface (district majoritairement francophone de Winnipeg), et que l'Ouest canadien est bien présent dans son œuvre. Enfin, le Manitoba connaît un établissement d'enseignement supérieur francophone, le Collège Universitaire de Saint-Boniface, et un quotidien de langue française, La Liberté de Saint-Boniface (publié dp. 1913; www. presseouest.mb.ca/).

La Colombie-Britannique entra dans la Confédération en 1871; quant aux provinces de la Saskatchewan et de l'Alberta, elles furent créées en 1905 à même les Territoires du Nord-Ouest. Aucune d'entre elles ne connaît l'équivalent de l'article 23 de l'Acte du Manitoba dans sa loi constitutive et le français n'y jouit d'un statut officiel que dans les institutions relevant du gouvernement fédéral. L'immigration francophone en provenance de l'est du pays y fut relativement tardive (à partir de 1890 env. pour l'Alberta, v. Rochet 1994:433) et numériquement faible en comparaison avec 
l'important flux migratoire que connut la Nouvelle-Angleterre (v. ci-dessous 1.5.). En Saskatchewan, 2,0\% des habitants déclarent le français comme langue maternelle, mais seulement $0,6 \%$ le parlent à la maison; le pourcentage de bilingues s'y élève à $5,2 \%$. Pour l'Alberta, ces pourcentages sont très semblables avec respectivement 2,0\%, 0,6\% et $6,7 \%$; en Colombie-Britannique, on relève $1,4 \%, 0,4 \%$ et $6,7 \%$ (v. tableaux $1 b, 2 b$ et 3 ). La minorité francophone d'Alberta réussit à publier un quotidien de langue française, $L e$ Franco d'Edmonton, depuis 1928 (www. compusmart.ab.ca/lefranco/). Le succès de la romancière d'expression française Nancy Huston, Parisienne d'adoption d'origine canadienne anglaise (Calgary, Alberta), rappelle que le français est encore, malgré tout, une langue de culture très appréciée dans certains cercles canadiens anglais.

\subsection{La Nouvelle-Angleterre.}

À partir des années 1840, l'excédent démographique des zones rurales québécoises et acadiennes commence à être absorbé par les états voisins de la NouvelleAngleterre (Maine, New Hampshire, Vermont, Massachusetts, Rhode-Island et Connecticut), dont l'industrialisation croissante nécessite de forts contingents de main-d'œuvre bon marché, en particulier dans le domaine des filatures. Au total, de 1840 à 1930, l'émigration canadienne-française en Nouvelle-Angleterre se serait élevée à env. 525000 personnes (Breton 1993:654). Ils forment vite une présence visible et organisée dans les petites agglomérations industrielles, où le système des «paroisses» catholiques est transplanté; leur grande cohésion sociale, ainsi qu'un certain mouvement de va-et-vient avec le Québec, assure la transmission et le maintien de la langue française jusqu'à la Seconde Guerre mondiale, à travers tout un ensemble d'institutions: écoles, églises, quotidiens, hôpitaux, orphelinats, bibliothèques, associations culturelles, récréatives ou syndicales. Les quartiers qu'ils occupent sont appelés «Petits Canadas» et la population franco-américaine de Nouvelle-
Angleterre constitue à l'époque un véritable «Québec d'en bas». Sur le plan linguistique, un aussi important mouvement de population ne pouvait manquer d'avoir des conséquences: comme le signale Claude Poirier (1994:89), la relative uniformité (quantitative et qualitative) des emprunts à l'anglais sur le territoire québécois s'explique sans doute par les nombreux déplacements entre les deux pays. Plusieurs anglicismes sont d'ailleurs attestés pour la première fois dans la presse franco-américaine, qui constitue une source précieuse pour la lexicologie historique du français québécois.

Dans la seconde moitié du $\mathrm{XX}^{\mathrm{e}}$ siècle, les vieilles structures urbaines héritées de la Révolution industrielle s'étiolent et la population francophone, jadis concentrée dans les centres-villes, se disperse çà et là dans les banlieues-dortoirs; la connaissance et la transmission du français reculent, les descendants des Québécois et des Acadiens se fondant tranquillement dans le creuset américain. L'abandon de la langue d'origine chez les immigrants de troisième et de quatrième génération aux États-Unis est d'ailleurs une constante qui s'observe chez presque tous les groupes ethniques (à l'exception des hispanophones). Au recensement de 1980 (v. Breton 1993:653), plus d'un million de personnes (1 191000) se déclaraient d'origine française en NouvelleAngleterre; 412000 d'entre elles affirmaient encore parler français au foyer. Ce sont en fait surtout des personnes âgées, la plupart des jeunes ayant perdu la possibilité de s'exprimer dans cette langue; quant à ceux qui la parlent, il s'agit souvent d'étudiants l'ayant réapprise au collège et à l'université, et désireux de renouer avec leurs origines. Au recensement de 1990, le nombre total de répondants ayant le français comme langue d'usage à la maison est tombé à env. 360000 (v. tableau 4 pour le détail par état); l'assimilation semble inéluctable.

Dans le domaine littéraire, on relève quelques romans dans la période 1875-1936 (v. Senécal 1994), mais le plus célèbre écrivain franco-américain n'a écrit, paradoxale- 
ment, qu'en anglais (à l'exception de quelques rares passages): il s'agit bien sûr de Jack Kerouac (1922-1969), un des principaux représentants du mouvement «beatnik».

\subsection{Saint-Pierre et Miquelon.}

L'archipel de Saint-Pierre et Miquelon, seul territoire nord-américain resté jusqu'à nos jours en possession de la France (avec le statut administratif de "communauté territoriale»), couvre une superficie de 242 $\mathrm{km}^{2}$ et compte environ 6200 habitants, tous francophones. Il est situé à une vingtaine de kilomètres au sud de Terre-Neuve, à proximité d'une des zones les plus poissonneuses du monde. C'est d'ailleurs en raison de cette situation avantageuse que l'archipel était déjà connu par les pêcheurs européens au moyen âge; en 1520, il est officiellement «découvert» par des Portugais, mais Jacques Cartier en prend possession au nom du Roi de France en 1536. À l'origine simple refuge pour les pêcheurs de morue des côtes de l'ouest français, il connaît à partir du XVII ${ }^{e}$ siècle les premières tentatives françaises d'installation permanente, mais les nombreux conflits avec l'Angleterre empêchent l'établissement d'une véritable colonie. $\mathrm{Au}$ XVIII ${ }^{\mathrm{e}}$ siècle, les Îles de Saint-Pierre et Miquelon, au gré des conflits, passent aux mains des Anglais à d'innombrables reprises; chaque fois, les habitants sont déportés, en Acadie ou même en France, et les installations détruites. Ce n'est vraiment qu'avec le traité de Paris de 1815 que la rétrocession des îles à la France devient définitive, assortie d'un droit de pêche sur les côtes de Terre-Neuve. Les habitants de l'époque, qui forment la base de la population actuelle, sont des Acadiens longtemps réfugiés en France ainsi que des pêcheurs normands, bretons et basques. Le contact avec la France n'ayant jamais été interrompu depuis, l'archipel a reçu des apports migratoires de la Métropole, qui y a régulièrement envoyé, en plus des pêcheurs, des fonctionnaires, des militaires et des religieux; d'autre part, les habitants de l'île ont entretenu des contacts avec le Canada, anglophone et francophone. Le français qu'on y parle aujourd'hui est unique: s'il se démarque peu du français métropolitain (en comparaison avec les autres variétés nordaméricaines), il s'en distingue toutefois par un certain nombre de diatopismes, surtout lexicaux, d'origine acadienne, laurentienne, terre-neuvienne (anglicismes) ou française (région de Saint-Malo-Granville); v. Brasseur / Chauveau 1990. Il n'est d'ailleurs pas toujours facile de déterminer par quelle voie un mot a pénétré l'usage (v. en particulier Chauveau 1992:201).

\subsection{Port-au-Port (Terre-Neuve).}

Il importe de distinguer l'histoire de la présence française à Terre-Neuve de celle de la langue encore parlée par les FrancoTerreneuviens, aujourd'hui concentrés dans la péninsule de Port-au-Port, sur la côte occidentale de l'île. Dès le XVI ${ }^{\mathrm{e}}$ siècle, des pêcheurs français fréquentent les côtes de Terre-Neuve, où le poisson abonde. Des navires séjournent dans les anses pendant la belle saison et des installations temporaires pour le traitement du poisson voient le jour sur la terre ferme. Au XVII ${ }^{e}$ siècle, des colons tentent de s'installer peu à peu d'une façon permanente; Plaisance est fondé en 1626, et fortifié en 1662. En 1687, un recensement révèle la présence de 660 Français sur la côte sud de Terre-Neuve, mais un conflit avec l'Angleterre mène à la destruction de Plaisance en 1690. Au début du XVIII ${ }^{\mathrm{e}}$ siècle, les guerres continuent de faire rage et par le Traité d'Utrecht (1713) la France perd Terre-Neuve au profit des Anglais, tout en conservant un droit de pêche dans la région. À l'issue de la Guerre de Sept Ans, la France perd le reste de ses possessions au Canada, mais ses droits de pêche à Terre-Neuve sont encore préservés. Alors que de 1713 à 1783, la côte dite «française» (c'est-à-dire où les pêcheurs français ont le droit de séjourner pour le traitement du poisson) est du côté oriental de l'île, à partir du Traité de Versailles c'est la côte occidentale qui est octroyée aux Français, jusqu'à ce qu'en 1904 la France abandonne entièrement 
ses droits de pêche à Terre-Neuve. Le peuplement francophone est composé à la base d'Acadiens réfugiés, qui constitueront les familles souches de la population franco-terreneuvienne. Viendront s'y joindre tout au long du $\mathrm{XIX}^{\mathrm{e}}$ siècle de nombreux pêcheurs originaires de France ou de Saint-Pierre et Miquelon, qui décident d'échapper à leur sort en s'installant de manière plus ou moins légale à Terre-Neuve, parmi la population francophone de la côte ouest, et plus spécialement dans la péninsule de Port-auPort. La présence française est très visible dans la toponymie des côtes de Terre-Neuve (v. Magord 1996:192-195 pour une liste nonexhaustive de 257 noms de lieux français) ainsi que, dans une moindre mesure, dans les patronymes (ibid. 196-198). L'étude des caractéristiques grammaticales et lexicales de la langue des Franco-Terreneuviens (v. Niederehe 1991 et Brasseur, à paraître) montre qu'il s'agit clairement d'une variété de français nord-américain, plus précisément acadien, et ce en dépit des apports migratoires du XIX $^{\mathrm{e}}$ siècle originaires de France; en effet, ces marins déserteurs et ces pêcheurs - du reste souvent partis au large se sont unis à des Acadiennes, et ce sont elles qui ont transmis leur langue aux descendants. Seule une douzaine de technicismes propres au vocabulaire de la pêche (v. Chauveau 1998:106), inconnus au Canada, proviennent indubitablement des côtes de Normandie et de Bretagne.

Terre-Neuve abrite la plus petite minorité francophone de toutes les provinces canadiennes; on y relève aujourd'hui (recensement 1996) 2275 personnes reconnaissant le français comme langue maternelle $(0,42 \%$ de la population totale), mais seulement 880 qui le parlent encore à la maison, dont env. 155 unilingues; toutefois, 21260 Terreneuviens affirment être bilingues (3,9\% du total). V. tableaux $1 b, 2 b$ et 3 .

\subsection{Aperçu statistique général.}

Pour l'ensemble du Canada, au dernier recensement (1996), 23,3\% des Canadiens ont déclaré le français comme langue maternelle (tableau 1a) et $22,3 \%$ comme langue d'usage à la maison (tableau 2a). Au recensement de 1981, 25,7\% des Canadiens avaient déclaré le français comme langue maternelle (v. Wolf 1987:60). En quinze ans, ce pourcentage a donc reculé mais en chiffres absolus l'accroissement est tout de même de 387565 locuteurs. 17,0\% des Canadiens se présentent comme bilingues anglais-français, et $14,3 \%$ comme francophones exclusifs, pour un total de $31,3 \%$ de personnes aptes à s'exprimer en français (8 920405 en chiffres absolus, v. tableau 3).

\section{Origines, substrats et adstrats.}

Les variétés de français parlées de nos jours au Canada (d'une part l'acadien, d'autre part le «laurentien» et ses extensions territoriales) reposent sur un fonds historique hérité des variétés de français normatif, populaire et régional du $\mathrm{XVII}^{\mathrm{e}}$ siècle et marqué par quelques traits dialectaux. L'adstrat amérindien n'a légué qu'un tout petit contingent de mots, contrairement à l'adstrat anglais qui a donné lieu à des centaines d'emprunts.

\subsection{Fonds historique; genèse; variation géographique.}

Le problème de la langue parlée par les premiers colons, et de l'établissement semble-t-il relativement précoce d'une koinè de français colonial en Nouvelle-France, a fait couler beaucoup d'encre ces dernières années. D'une part, les témoignages d'époque (et, indirectement, la nature du français québécois et acadien actuel) montrent que la langue parlée par les «habitants» à l'époque coloniale était bien du français, et non un patois ou un mélange de dialectes d'oïl; d'autre part, les patois étaient alors encore très vivants dans toute la France. Cette situation en apparence contradictoire a amené les chercheurs à se demander comment la koinè initiale s'était formée. Les premiers colons ont-ils apporté avec eux leurs patois, qu'ils auraient peu à peu aban- 
donnés au profit du français à la faveur de mariages mixtes et en raison du prestige de la langue des élites, ou ont-ils directement transplanté au Canada leur propre variété de français, populaire et régional? Il est généralement admis aujourd'hui par la communauté scientifique que la grande majorité des sujets venus s'installer dans la colonie laurentienne au XVII siècle connaissaient bien le français, et ce pour les raisons suivantes: «1) les colons originaires du domaine d'oc formaient un groupe très marginal; 2) les colons venaient en majorité des villes; 3 ) ils appartenaient en majorité à la catégorie des travailleurs mobiles (journaliers et gens de métier) $[. .$.$] ; 4) près de la moitié d'entre eux$ étaient alphabétisés; 5) leurs congénères acadiens [qui bénéficièrent moins longtemps de l'exemple des élites et venaient en grande partie de régions réputées patoisantes] parlaient français.» (Mougeon / Beniak 1994:8-9). Dans de telles circonstances, il n'y a pas lieu de s'étonner que les patois soient rapidement disparus - si tant est qu'ils ont jamais eu cours - en Nouvelle-France.

Il convient toutefois de se demander quelle est la nature exacte du (ou des) français qui ont contribué à la genèse des français canadiens, et de quelle façon celle-ci s'est réalisée. Les réponses à ces questions sont encore partielles et insatisfaisantes, car on connaît mal la situation sociolinguistique qui régnait alors en France. Il semble raisonnable de postuler que trois variétés ont contribué, à des degrés divers, à la formation de la koinè initiale: les français régionaux de l'ouest et du centre du domaine d'oïl, le vulgaire parisien, et la variété de prestige (issue historiquement de l'acrolecte parisien, mais déjà devenue un sociolecte supra-régional à l'époque de la colonisation). Seul un alignement précoce sur cette dernière peut expliquer, par exemple, la quasi-absence de la terminaison -iau (pour -eau) en francoquébécois, alors qu'elle est (ou était) très répandue dans nombre de français régionaux, en Île-de-France même (Morin 1994:209 sqq.). Le québécois ne connaît guère le ouisme (houme pour homme, etc.), les formes verbales de $1^{\text {re }}$ pers. du pluriel en je (je sons, etc.) et de $3^{\mathrm{e}}$ pers. du pluriel en -ont (ils parlont, etc.), phénomènes typiques de l'acadien, fréquents en français pré-classique, et qui pourraient aussi avoir été abandonnés en raison de la pression normative exercée par l'appareil administratif colonial, dont l'influence s'est fait sentir plus longtemps et plus profondément dans la vallée du SaintLaurent qu'en Acadie. L'alignement sur la norme de l'époque n'a d'ailleurs pas toujours été complet: les différentes formes du semiauxiliaire du futur proche encore en usage au Québec en témoignent (vas, m'as, vais, v. Mougeon 1996). En outre, certains phénomènes divergents par rapport au français de France sont nés et se sont répandus de façon tout à fait indépendante, vraisemblablement pour des raisons structurales internes, comme cela semble être le cas de la diphtongaison (v. Dagenais 1993). Enfin, on relève des phénomènes de convergence structurale postérieurs à la Conquête, comme la fermeture du $o$ ouvert final (dans des mots comme croc, sot, trop), qui s'est produite au $\mathrm{XIX}^{\mathrm{e}}$ siècle de manière parallèle et indépendante au Québec et dans la norme parisienne, ou la perte des oppositions de durée vocalique en fin de mot. Cette situation amène à se poser la question suivante: «Serait-elle [la perte des oppositions de durée vocalique] liée à l'hétérogénéité linguistique initiale aussi bien des colons que des classes dominantes parisiennes, hétérogénéité qui favoriserait certains types de changements, alors que la stabilité sociale et géographique des populations francophones d'Europe, même dans le voisinage de Paris, jusqu'au début du $\mathrm{XX}^{\mathrm{e}}$ siècle serait un facteur de conservatisme linguistique?» (Morin 1994:231).

Il faudrait encore pouvoir expliquer la variation diatopique à l'intérieur même du québécois et de l'acadien. Québec et Montréal semblent avoir agi comme centres directeurs (v. entre autres Verreault / Lavoie 1996) et contribué à la diffusion de nombreux régionalismes, respectivement typiques de l'est et de l'ouest de la province (cf. par ex. la réalisation resp. vélaire [Québec] et apicale [Montréal] du $r$ ), mais d'autres phé- 
nomènes tels l'affrication partielle ou inexistante relevée dans certaines zones de Charlevoix (Poirier 1994:79-82) échappent à cette dichotomie. Pour l'Acadie, il convient de se demander si la variation diatopique observée de nos jours remonte à l'époque de la colonisation ou si son apparition est postérieure. D'après Flikeid 1994: 320, «une unité linguistique considérable a dû régner dans l'ancienne Acadie, quelle que soit son origine», mais la co-existence de certaines variantes a pu par la suite déboucher, par focalisation, sur des cas de variation, phénomène favorisé par l'éclatement de la population en plusieurs groupes relativement isolés, au gré des aléas de l'histoire. La région de la Baie Sainte-Marie, au sud-ouest de la Nouvelle-Écosse, serait la plus ancienne zone de peuplement continu et une des plus archaïsantes; les villages acadiens du nord-est de la Nouvelle-Écosse auraient quant à eux subi plus longtemps (tout au long du XVIII ${ }^{\mathrm{e}}$ siècle) des influences externes (France, Saint-Pierre et Miquelon, Québec); quant au Nouveau-Brunswick, l'influence de la norme et du français québécois y a été plus forte qu'ailleurs.

\subsection{Substrat dialectal.}

Le français régional importé par les colons était plus ou moins marqué par le contact avec les patois de l'ouest et du centre du domaine d'oïl. Si cette influence n'a guère laissé de traces dans la phonologie, la morphologie et la syntaxe, le vocabulaire en revanche a retenu un certain nombre d'unités lexicales empruntées à divers patois d'oïl (sous des formes éventuellement francisées). Cette situation se présente en particulier dans des champs sémantiques (travaux des champs, menuiserie, flore ou faune locale, etc.) où la langue de la cour ne disposait pas des ressources lexicales nécessaires, ou n'avait pas encore réussi à les diffuser. Certains de ces types lexicaux couvraient de très grandes étendues; on ne s'étonne donc pas de leur survivance en franco-canadien, mais de tels mots ne permettent pas de déterminer avec précision l'origine des colons res- ponsables de leur diffusion. Il est cependant parfois possible, par le recoupement des aires identifiées, de repérer à quelques kilomètres près l'origine des sujets dont l'influence fut déterminante dans l'aménagement terminologique d'un champ sémantique donné. Il a ainsi pu être démontré (Chauveau 1993, 1995 et à paraître; Chauveau/Lavoie 1993, 1996) que les Percherons ont réussi à diffuser - en particulier dans l'Est québécois - bon nombre de termes typiques du vocabulaire agricole de leur région, même s'ils ne représentaient qu'un tout petit pourcentage de la population totale venue s'établir en Nouvelle-France pendant le régime français, et ce en raison de plusieurs facteurs: plus nombreux dans la première phase de la colonisation et venus en familles, ils ont eu plus de descendants que les colons installés par la suite; agriculteurs venus exprès pour cultiver la terre, c'est vraisemblablement auprès d'eux que leurs compatriotes originaires des villes ont appris le travail de la terre, et le lexique spécialisé s'y rapportant.

\subsection{Adstrat amérindien.}

Les français laurentien et acadien n'ont guère été influencés par les adstrats amérindiens; ni la phonétique, ni la grammaire n'en ont retenu la moindre trace. Seul le lexique témoigne du contact entre les communautés, mais en additionnant tous les types lexicaux relevés au Canada du $\mathrm{XVI}^{\mathrm{e}}$ siècle à nos jours, archaïsmes et régionalismes inclus, on ne dépasse pas la centaine d'unités (Faribault 1993:210; les toponymes d'origine amérindienne sont, en revanche, très nombreux). Qui plus est, certains de ces emprunts existant aussi en français de France, on pourrait être tenté de croire qu'ils n'ont rien de spécifiquement canadien; toutefois, un certain nombre de différences s'observe: «a) les emprunts connaissent rarement des glissements de sens [au Canada], à l'opposé de ce qui se passe en français européen; b) l'intégration au système de la langue est plus poussée qu'en français européen, puisqu'on trouve des 
dérivés morphologiques; c) on observe une variation de l'usage sur le territoire du français canadien; d) l'emprunt se fait par contact direct et de façon continue, à partir du XVIe siècle jusqu'à récemment; e) le phénomène est marginal, par rapport à l'emprunt anglais beaucoup plus important, mais tout de même plus marqué qu'en français européen.» (Faribault 1993:205). Outre les appellatifs et les toponymes, il ne faut pas oublier la catégorie intermédiaire des gentilés (ou ethniques), traités en profondeur par S. Canac-Marquis dans le Dictionnaire historique du français québécois (= Poirier 1998; cf. abénakis, esquimau, inuit, iroquois, iroquoien, etc.).

\subsection{Adstrat anglais et anglo-américain.}

Les premiers emprunts à l'anglais apparaissent dans des textes d'archives quelques années à peine après la Conquête (v. cidessus 1.2.3.). Juneau 1969:34 en relève déjà une douzaine de 1771 à la fin du XVIII ${ }^{\mathrm{e}}$ siècle; ils désignent des objets de civilisation introduits par les Anglais. Au XIX ${ }^{\mathrm{e}}$ siècle, ce type d'emprunts augmente au gré des échanges commerciaux et des innovations technologiques, mais les calques (sémantiques et phraséologiques) font vite leur apparition; ils sont dus au statut de «langue de traduction» désormais réservé au français dans la presse, la publicité et les textes de loi, mais aussi aux contacts entre groupes linguistiques et à l'émergence du bilinguisme chez les élites francophones. Dans la seconde moitié du $\mathrm{XIX}^{\mathrm{e}}$ siècle, la naissance d'un prolétariat urbain ainsi que les mouvements de populations entre le Québec et la NouvelleAngleterre (v. ci-dessus 1.5.) étendront le phénomène à toutes les classes de la société, dans toutes les régions francophones du pays. L'importance de la réaction à l'anglicisme est proportionnelle à l'envergure du phénomène: dès les tout premiers recueils de français correctif publiés au Canada (tel Viger 1810; v. Poirier 1978:43), les divers types d'emprunts à l'anglais sont la cible privilégiée des gardiens du bon usage. La tradition des listes d'anglicismes à éviter se perpétue jusqu'à nos jours (v. Poirier 1978:101-106 pour une bibliographie) et tend à surévaluer l'importance de ces emprunts en français québécois; plusieurs d'entre eux sont d'ailleurs tombés en désuétude depuis la Révolution tranquille. Le Dictionnaire de fréquence des mots du français parlé au Québec (Beauchemin et al. 1992) révèle que $6 \%$ des types lexicaux du corpus sont des emprunts lexématiques à l'anglais, mais ils ne représentent que $0,28 \%$ des occurrences dans le discours. Il faut toutefois spécifier que cette source ne tient pas compte des calques, ce qui fausse les résultats; la langue de la presse est particulièrement marquée par ce type d'anglicismes, qui passent plus facilement inaperçus (v. Poirier 1978:81). D'une manière générale, les publications qui traitent des anglicismes sont d'une qualité médiocre. Sans profondeur historique, elles interprètent à tort certaines survivances galloromanes comme des emprunts à l'anglais; en revanche, d'innombrables calques leur échappent. En outre, elles ne rendent pas compte de la dimension culturelle attachée à ces mots. On ne dispose à l'heure actuelle d'aucun dictionnaire philologique et historique fiable sur les anglicismes en franco-québécois; on consultera toutefois avec profit DFQPrés (= Poirier 1985) et DHFQ (= Poirier 1998) pour un modèle de traitement lexicographique des anglicismes qui mériterait de faire école.

Le contact avec l'anglais n'a guère exercé d'influence sur la phonétique du franco-canadien (sauf bien sûr dans les régions où le français, très minoritaire, est en voie de disparition; on notera aussi la présence du $r$ anglais dans quelques zones de contact, v. Dulong/Bergeron 1980, t. 1, p. 31). On remarque toutefois que les mots anglais ont davantage tendance à être prononcés «à l'anglaise» qu'en France, au point que le français québécois connaît aujourd'hui des oppositions phonologiques originales inédites dans le standard européen (pool $\neq$ poule; beat $\neq$ bitte); ce phénomène est toutefois assez récent et n'apparaît pas dans les emprunts plus anciens (bean se prononce avec un $i$ bref relâché). Dans d'autres cas, l'adaptation des sons de l'anglais aux habitudes articulatoires des Québécois débouche sur un résultat qui ne correspond pas à celui 
que l'on observe chez des sujets français, sans qu'il soit facile de proposer une explication satisfaisante à cette situation; Auger 1990 remarque par exemple que le $t h$ anglais (resp. [ $\theta]$ ou [ $\delta]$ selon qu'il est sourd ou sonore) est souvent rendu par les sifflantes $[s]$ et $[z]$ chez des sujets français, mais par les occlusives $[t]$ et $[d]$ chez des Québécois.

Il importe de signaler en terminant que les échanges entre l'anglais et le français au Canada n'ont pas toujours été à sens unique. L'anglais canadien (et nordaméricain) a emprunté un certain nombre de termes au français canadien, en particulier dans les domaines de la flore, de la faune et de la topographie (les traces en sont encore très visibles dans la toponymie), et ce par l'entremise des «voyageurs», «engagés» et autres «coureurs de bois» (v. DHFQ) impliqués dans le commerce des fourrures et agissant souvent à titre d'intermédiaires entre les Amérindiens et les commerçants britanniques (v. Vézina 1993 et à paraître). Encore de nos jours, l'anglais montréalais se distingue par certains emprunts au français général et au franco-québécois (metro "subway", polyvalent school "high school", etc.).

\section{Les études linguistiques.}

Le français canadien est l'une des variétés les plus étudiées du monde francophone; la production des dix ou quinze dernières années n'a fait que renforcer cette situation. Dans le domaine de la géographie linguistique, l'Atlas linguistique de l'est du Canada, de G. Dulong et G. Bergeron (1980), a été complété en 1985 par Les parlers français de Charlevoix, du Saguenay, du Lac Saint-Jean et de la Côte-Nord de Th. Lavoie et al. (1985) et plus récemment par l'Atlas linguistique du vocabulaire maritime acadien de L. Péronnet et al. (1998). À ces riches cueillettes de données brutes sont venus s'ajouter des articles monographiques où les matériaux sont soumis à une approche explicative (v. Chauveau 1993, 1995 et à paraître; Chauveau / Lavoie 1993, Chauveau / Lavoie 1996, Verreault / Lavoie 1996). En lexicographie et lexicologie, la moisson n'est pas moindre. Pour ce qui est des ouvrages différentiels, on citera d'abord le volume de présentation du Dictionnaire $d u$ français québécois du TLFQ (Poirier 1985), méthodologiquement exemplaire, et qui annonçait le Dictionnaire historique $d u$ français québécois, dont un premier tome est paru récemment (Poirier 1998). Les études de lexicologie bénéficient de la nouvelle édition (Gérin 1993) du glossaire acadien de Pascal Poirier (1927-1933), naguère peu accessible, ainsi que du travail de P. W. Halford (1994) qui met à la disposition des chercheurs les richesses des Façons de parler du père Potier (1743-1758; v. ci-dessus 1.3.). L'excellent Dictionnaire des régionalismes de Saint-Pierre et Miquelon de P. Brasseur et J.-P. Chauveau (1990), qui sera bientôt suivi d'un ouvrage équivalent pour le français de Terre-Neuve (P. Brasseur, en préparation), est venu apporter un précieux complément à notre connaissance des français nordaméricains. Enfin, Yves Cormier, de l'Université Sainte-Anne (Baie Sainte-Marie, Nouvelle-Écosse), travaille à la rédaction d'un nouveau dictionnaire historicocomparatif d'acadianismes. Quant à la lexicographie non différentielle, il faut noter la parution du Dictionnaire du français plus (Poirier 1988) et du Dictionnaire québécois d'aujourd'hui (Boulanger 1992), qui se situent tous les deux dans la foulée du Bélisle (1957, 1979). Les études de lexicologie franco-canadienne bénéficient désormais d'un dictionnaire de fréquence (Beauchemin et al. 1992), d'un recueil de premières attestations (Rézeau 1998), et d'un imposant répertoire de données métalexicales élaboré au TLFQ (Trésor de la langue française au Québec, Université Laval) par Louis Mercier, appelé Index lexicologique québécois (v. Poirier 1998:xxxvii-xxxviii). En anthroponymie, le Dictionnaire généalogique des familles du Québec (Jetté 1983) rendra service aux chercheurs; la toponymie est prise en charge par le Répertoire toponymique du Québec (1987) et le recueil Noms et lieux du Québec (1994; v. encore Lapierre 1994 pour les toponymes d'origine française hors Québec). Pour les détopony- 
mes, cf. le Répertoire des gentilés du Québec (Dugas 1987). En grammaire, de nombreux articles monographiques paraissent régulièrement mais les synthèses sont rares, à l'exception de la Grammaire québécoise d'aujourd'hui de J.-M. Léard (1995). L'absence d'une synthèse en phonétique se fait également sentir face au foisonnement des publications spécialisées, mais l'on consultera avec profit le volet «Phonétique» du site Internet du CIRAL (Centre international de recherches en aménagement linguistique) de l'Université Laval (www. ciral.ulaval.ca) pour une présentation synchronique; pour la diachronie, cf. entre autres Dagenais 1986 et Morin 1991, 1994 et 1996. Juneau 1972 reste un classique pour la période coloniale. Enfin, le corpus littéraire est richement servi par le monumental Dictionnaire des auvres littéraires du Québec (DOLQ), dont 6 tomes sont parus à ce jour.

\section{Le problème de la norme.}

Jusqu'à la Conquête (v. ci-dessus 1.2.3.), la langue parlée dans la vallée laurentienne est une variété de français assez tôt unifiée (v. ci-dessus 2.1.), objet de jugements appréciatifs favorables de la part des Européens de passage. Tout au plus remarque-ton, à la fin du régime français, quelques dizaines d'amérindianismes ainsi qu'un contingent de mots pouvant être interprétés $a$ posteriori comme des archaïsmes, des régionalismes ou des innovations (soigneusement notés par le père Potier, v. Halford 1994:217-304). Sur le français acadien de l'époque, on ne sait pas grandchose; tout au plus peut-on constater que le contact avec la métropole et l'influence linguistique des classes dirigeantes ont cessé d'agir un demi-siècle plus tôt qu'au «Canada». La Conquête ayant comme conséquences la coupure des liens avec la France, le départ d'une partie des élites et une situation de subordination fonctionnelle du français face à l'anglais, la langue évoluera désormais d'une façon indépendante et partiellement divergente. $\mathrm{Au} \mathrm{XIX}^{\mathrm{e}}$ siècle, les premiers juge- ments dépréciatifs commencent à apparaître; ils visent surtout les anglicismes, qui dans certains champs sémantiques se sont multipliés, mais aussi nombre de prononciations, mots et tournures issus du français populaire ou rural et stigmatisés par les observateurs en raison de leur nonadéquation à la norme écrite transmise par les dictionnaires et les manuels de grammaire de l'époque. La quasi-absence de stratification sociale au sein d'une population aux origines globalement très humbles, combinée à un analphabétisme dominant, explique la diffusion généralisée de tels emplois, que la norme européenne postrévolutionnaire et républicaine n'a pas retenus. $\mathrm{Au} \mathrm{XX}^{\mathrm{e}}$ siècle, alors que les contacts directs avec la France reprennent lentement (disques, radio, tournées d'artistes européens au Canada, études ou voyages outre-mer, conflits mondiaux, cinéma, télévision, etc.), les élites prennent douloureusement conscience du caractère divergent du français canadien, même celui des classes cultivées, face à la norme européenne; elles en concevront un certain malaise, qui ne s'est pas vraiment estompé jusqu'à nos jours, malgré certaines évolutions sociales.

Il convient de distinguer, grosso modo, deux acceptions du mot «norme»: d'une part, celle que l'on pourrait appeler «implicite», «objective» ou «sociale» et qui existe par défaut, sans être consignée par écrit; d'autre part, la norme explicite et prescriptive des ouvrages de référence (grammaires, dictionnaires). Par définition, le français laurentien a toujours connu une norme implicite, dotée de plusieurs niveaux de langue adaptés aux différentes situations de communication, et qui a du reste sensiblement évolué au cours des dernières décennies du $\mathrm{XX}^{\mathrm{e}}$ siècle ( $\mathrm{v}$. Gendron 1987, 1990). Le problème réside dans la distance existant entre la norme implicite et la norme prescriptive (situation perçue comme anormale dans le monde francophone) et sur les moyens qu'il faut prendre pour l'éliminer ou l'atténuer. Schématiquement, deux écoles s'affrontent: les uns voudraient, sans grand réalisme, que l'usage québécois s'aligne entièrement sur la 
norme prescriptive; d'autres aimeraient rapatrier cette norme et la plier à l'usage spontané. Les partisans de cette seconde approche n'ont cependant pas toujours les moyens de leurs ambitions, cette démarche impliquant une description scientifique approfondie de la norme implicite sous tous ses aspects (phonétiques, grammaticaux et lexicaux, sur les axes diachronique, diatopique, diastratique et diaphasique). La réalité observable se situe à mi-chemin entre ces deux pôles. Le franco-québécois a abandonné depuis la Révolution tranquille (v. ci-dessus 1.2.4.) plusieurs traits archaïques, populaires et ruraux ainsi que de nombreux anglicismes, grâce aux effets combinés de l'urbanisation, de la démocratisation de l'enseignement, de l'influence des médias électroniques mais aussi sans doute de la pression puriste et des campagnes de francisation (entreprises, commerces, affichage); cela dit, les productions langagières des Québécois (presse, littérature, spectacle, droit, politique, administration, etc.) n'en conservent pas moins une personnalité propre, ce qui va de soi et ne choque personne. Dans les domaines délicats de la féminisation des titres et des emprunts à l'anglais, les instances officielles ont même clairement pris leurs distances face à la France. Cette volonté d'indépendance est cependant encore très limitée. La lexicographie (descriptive) québécoise a produit ces dernières années deux ouvrages non contrastifs, le Dictionnaire du français plus (DFPlus = Poirier 1988) et le Dictionnaire québécois d'aujourd'hui (DQA = Boulanger 1992). Ceux-ci partagent une caractéristique structurelle tout à fait inédite: les mots québécois n'y sont pas marqués comme tels, alors que les mots considérés par les auteurs comme des «francismes» (sur les problèmes théoriques soulevés par ce concept et son application en lexicographie, v. Verreault 1996) reçoivent la marque «France». Les artisans du DFplus, conscients de la difficulté de faire accepter au public un tel dictionnaire, ont volontairement choisi de ne retenir à la nomenclature que des québécismes neutres, mettant de côté les mots traditionnellement stigmatisés. L'absence de marquage diatopique pour les québécismes leur fut reproché, mais dans l'ensemble les choix de nomenclature ne choquèrent pas l'opinion (à l'exception de certains linguistes partisans de la norme québécoise qui auraient préféré, un peu naïvement comme on le verra ci-dessous, que les mots québécois les plus fréquents soient tous pris en charge, indépendamment de leur position sur l'axe diastratique). La réception du DQA fut plus mouvementée. L'ouvrage comporte des québécismes relevant de tous les niveaux de langue et donc d'innombrables emplois considérés par une bonne partie des élites comme de simples «fautes de langage» qu'on doit s'appliquer à éliminer (indépendamment du fait que ces emplois sont tous très fréquents à l'oral et même à l'écrit, dans la littérature et de plus en plus dans la presse, chez des chroniqueurs ayant intégré à leur langue écrite plusieurs québécismes courants de la langue parlée). La réaction fut quasiunanime: le DQA fit l'objet de jugements extrêmement sévères de la part de nombreux intervenants (non-linguistes) qui ne sont pas du tout prêts à accepter que la norme prescriptive euro-centriste soit remplacée par une norme auto-référentielle coïncidant avec l'usage; v. Boisvert et al. 1993 pour un relevé des réactions, souvent exacerbées, soulevées par la parution du DQA. Le débat sur la norme au Québec est plus virulent que jamais; il a été entretenu ces dernières années par la publication de nombreux pamphlets et articles polémiques dans lesquels les partisans des deux écoles s'affrontent dans un véritable dialogue de sourds.

Si plusieurs Québécois ressentent un sentiment d'infériorité face à la France et à l'Amérique anglo-saxonne, en Acadie la situation est compliquée par l'existence d'un complexe d'infériorité envers les Québécois, en position de force au sein de la francophonie canadienne. Les produits dictionnairiques élaborés au Québec sont d'ailleurs insatisfaisants du point de vue acadien (v. Gérin 1998). Le français des Acadiens a connu des évolutions divergentes ces dernières décennies (v. Flikeid 1994, Péronnet 1996): si dans certaines régions il 
devient de plus en plus anglicisé, archaïque et fragmentaire, dans d'autres il s'est en revanche rapproché de la norme prescriptive. On notera en outre un phénomène qui ne date pas d'hier mais qui connaît une ampleur croissante, l'emprunt au français québécois, diffusé massivement par la télévision.

\section{Bibliographie sélective.}

\subsection{Répertoires bibliographiques.}

Anctil, Pierre, A Franco-American Bibliography. New England, Bedford (N. H.), National Materials Development Center, 1979.

Centre d'études acadiennes, Inventaire général des sources documentaires sur les Acadiens, Moncton (N.-B.), Les Éditions d'Acadie, 1975-1977.

Dulong, Gaston, Bibliographie linguistique du Canada français de James Geddes et Adjutor Rivard (1906) continuée par Gaston Dulong, Paris, Klincksieck / Québec, Presses de l'Université Laval, 1966 (Bibliothèque française et romane, Série E: langue et littérature française au Canada 1).

Sabourin, Conrad F. / Lamarche, Rolande M., Le français québécois (Bibliographie analytique), Montréal, Office de la langue française, 1979.

Sabourin, Conrad F. / Lamarche, Rolande M., La francité canadienne, Montréal, Université de Montréal, 1985.

\subsection{Ouvrages de référence.}

Beauchemin, Normand / Martel, Pierre / Théoret, Michel, Dictionnaire de fréquence des mots du français parlé au Québec : fréquence, dispersion, usage, écart réduit, New York, Peter Lang, 1992.
Bélisle, Louis-Alexandre, Dictionnaire général de la langue française au Canada, Québec, Bélisle éditeur, 1957 (réédité en 1971 et en 1974).

Bélisle, Louis-Alexandre, Dictionnaire nordaméricain de la langue française, Montréal, Beauchemin, 1979.

Boulanger, Jean-Claude (dir.), Dictionnaire québécois d'aujourd'hui. Langue française, histoire, géographie, culture générale, rédaction dirigée par Jean-Claude Boulanger, supervisée par Alain Rey, Saint-Laurent (Québec), Dicorobert inc., 1992. Complété par un Atlas géographique \& historique, une Chronologie, un Dictionnaire de noms propres des des Annexes grammaticales.

Brasseur, Patrice / Chauveau, Jean-Paul, Dictionnaire des régionalismes de SaintPierre et Miquelon, Tübingen, Niemeyer, 1990 (Canadiana Romanica 5).

DHFQ: v. Poirier 1998.

DOLQ: Dictionnaire des auvres littéraires du Québec, sous la dir. de Maurice Lemire, t. 1, Des origines à 1900, avec la collab. de Jacques Blais, Nive Voisine et Jean Du Berger, Montréal, Fides, 1978, 2e éd., 1980; t. 2, 1900 1939, avec la collab. de Gilles Dorion, André Gaulin et Alonzo Le Blanc, 1980; t. 3, 1940-1959, avec la collab. de Gilles Dorion, André Gaulin, Alonzo Le Blanc et de Aurélien Boivin, Roger Chamberland, Kenneth Landry et Lucie Robert, 1982; t. 4, 1960-1969, 1984; t. 5, 1970-1975, avec la collab. de Aurélien Boivin, Gilles Dorion, André Gaulin, Alonzo Le Blanc, et de Roger Chamberland, Marie-José Des Rivières, Kenneth Landry, Michel Lord et Lucie Robert, 1987; t. 6, 1976-1980, sous la dir. de Gilles Dorion, avec la collab. de Aurélien Boivin, Roger Chamberland 
et Gilles Girard, 1994; en cours de publication.

Dugas, Jean-Yves, Répertoire des gentilés du Québec, Gouvernement du Québec, Commission de toponymie, 1987.

Dulong, Gaston / Bergeron, Gaston, Le parler populaire du Québec et de ses régions voisines. Atlas linguistique de l'Est du Canada. Gouvernement du Québec, Ministère des Communications en coproduction avec l'Office de la langue française, 1980, 10 vol.

Gérin, Pierre M. (éd.). Poirier, Pascal, Le glossaire acadien, Édition critique établie par Pierre M. Gérin, Moncton, Les Éditions d'Acadie, 1993 [éd. de l'original de 1927-1933].

Halford, Peter W., Le français des Canadiens à la veille de la Conquête. Témoignage du père Pierre Philippe Potier, s. j., Les Presses de l'Université d'Ottawa, 1994 (collection Amérique française 2).

Jetté, R. Dictionnaire généalogique des familles du Québec - des origines à 1730, Montréal, Presses de l'Université de Montréal, 1983.

Lavoie, Thomas / Bergeron, Gaston / Côté, Michelle, Les parlers français de Charlevoix, du Saguenay, du Lac Saint-Jean et de la Côte-Nord, Gouvernement du Québec, Office de la langue française Les Publications du Québec, 1985, 5 vol.

Léard, Jean-Marcel, Grammaire québécoise d'aujourd'hui. Comprendre les québécismes, Montréal, Guérin, 1995.

Massignon, Geneviève, Les parlers français d'Acadie, Enquête linguistique, Paris, Klincksieck, [1962]. 2 tomes.
Noms et Lieux du Québec, Dictionnaire illustré réalisé par la Commission de Toponymie du Québec, Sainte-Foy (Québec), Publications du Québec, 1994.

Péronnet, Louise / Babitch, Rose Mary / Cichocki, Wladyslaw / Brasseur, Patrice, Atlas linguistique du vocabulaire maritime acadien, Sainte-Foy (Québec), Les Presses de l'Université Laval, 1998 (collection Langue française en Amérique du Nord).

Poirier, Claude (dir.), Trésor de la langue française au Québec (TLFQ), Dictionnaire $d u$ français québécois. Description et histoire des régionalismes en usage au Québec depuis l'époque de la Nouvelle-France jusqu'à nos jours incluant un aperçu de leur extension dans les provinces canadiennes limitrophes, Volume de présentation, Sainte-Foy (Québec), Les Presses de l'Université Laval, 1985.

Poirier, Claude (rédacteur principal), Dictionnaire du français plus. À l'usage des francophones d'Amérique, Montréal, Centre éducatif et culturel Inc., 1988.

Poirier, Claude (dir.), Trésor de la Langue Française au Québec, Dictionnaire historique du français québécois. Monographies lexicographiques de québécismes, Sainte-Foy (Québec), Les Presses de 1’Université Laval, 1998.

Répertoire toponymique du Québec 1987, Gouvernement du Québec, Commission de toponymie, 1987.

Rézeau, Pierre (dir.), Datations et documents lexicographiques 48, Matériaux pour l'histoire du vocabulaire français rassemblés par l'équipe du Trésor de la Langue Française au Québec (TLFQ), Paris, C.N.R.S./Klincksieck, 1998. 
Société du parler français au Canada, Glossaire $d u$ parler français au Canada, contenant: $1^{\circ}$ les mots et locutions en usage dans le parler de la province de Québec et qui ne sont pas admis dans le français d'école; $2^{\circ}$ la définition et leurs différents sens, avec des exemples; $3^{\circ}$ des notes sur leur provenance ; $4^{\circ}$ la prononciation figurée des mots étudiés, Sainte-Foy (Québec), Les Presses de l'Université Laval, 1968 (reprint de l'original de 1930).

\subsection{Articles et monographies.}

Auger, Julie, "Two French accents in English - with special reference to metalinguistic awareness", dans Burmeister \& Rounds (éd.), Variability in second language acquisition (SLRF 90), Department of Linguistics, University of Oregon, 1990, p. 133150.

Boisvert, Lionel / Boulanger, Jean-Claude / Deshaies, Denise / Duchesneau, Lise, "Le dictionnaire comme révélateur d'insécurité linguistique", dans $M$. Francard (éd.), L'Insécurité linguistique dans les communautés francophones périphériques, Actes du colloque de Louvain-la-Neuve, 10-12 novembre 1993, Louvain-la-Neuve, 1993, vol. I, p. 187-198 (Cahiers de l'Institut de Linguistique de Louvain 19).

Bollée, Annegret, "Frankophonie IV. Regionale Varianten des Französischen außerhalb Europas I. a) Kanada b) Vereinigte Staaten und Karibik", dans Günter Holtus, Michael Metzeltin, Christian Schmitt (éditeurs), Lexikon der Romanistischen Linguistik (LRL), Tübingen, Niemeyer, 1990, Band V,1 (Französisch), p. 740-767.
Bouchard, Gérard / Tremblay, Marc, "Le peuplement francophone au Canada: survol historique et géographique (17 $\mathrm{e}_{-}$ 20 e siècle)", dans Gauthier / Lavoie 1995, p. 309-343.

Brasseur, Patrice, "Changements vocaliques initiaux dans le français de Terre-Neuve", dans Thomas Lavoie (éd.), Français du Canada - Français de France, Actes du quatrième Colloque international de Chicoutimi, Québec, du 21 au 24 septembre 1994, Tübingen, Niemeyer, 1996, p. 295-320 (Canadiana romanica 12).

Brault, Gérard-J., "Le français en NouvelleAngleterre", dans A. Valdman (dir.), Le français hors de France, avec la collaboration de R. Chaudenson et G. Manessy, Paris, Champion, 1979, p. 75-91.

Breton, Roland J.-L., "Crépuscule ou survivance des Francos et de la franco-américanie? (Une communauté bien vivante, mais qui ne pouvait pas être un pays", dans Didier de Robillard et Michel Beniamino (éditeurs), Le français dans l'espace francophone, Paris, Champion, 1993, t. II, p. 651663.

Castonguay, Charles, “Évolution de l'anglicisation des francophones au NouveauBrunswick, 1971-1991", dans Les Acadiens et leur(s) langue(s): quand le français est minoritaire, Actes du colloque sous la direction de Lise Dubois et Annette Boudreau, Centre de recherche en linguistique appliquée, Université de Moncton, Les Éditions d'Acadie, 1996, p. 47-62.

Charbonneau, Hubert / Guillemette, André, "Provinces et habitats d'origine des pionniers de la vallée laurentienne", dans Poirier 1994, p. 157-183. 
Chaudenson, Robert / Mougeon, Raymond / Beniak, Édouard, Vers une approche panlectale de la variation du français, Institut d'Études Créoles et Francophones, Université de Provence, Diffusion Didier Érudition, 1993 [v. en particulier "Variation en situation de contact linguistique: le français ontarien", p. 49-78].

Chauveau, Jean-Paul, "Saint-Pierre et Miquelon entre le Canada et la France", dans Hans-Josef Niederehe et Lothar Wolf (éditeurs), Français du Canada - Français de France. Actes du Colloque de Trèves du 26 au 28 septembre 1985, Tübingen, Niemeyer, 1987, p. 109-128 (Canadiana romanica 1).

Chauveau, Jean-Paul, "Le français à SaintPierre et Miquelon", dans Cahiers de lexicologie 61, 1992, p. 193-217.

Chauveau, Jean-Paul, "Le vocabulaire du matériel agricole au Canada et dans l'Ouest de la France", dans Hans-Josef Niederehe et Lothar Wolf (éditeurs), Français du Canada - Français de France. Actes du troisième Colloque international d'Augsbourg du 13 aи 17 mai 1991, Tübingen, Niemeyer, 1993, p. 73-107 (Canadiana romanica 7).

Chauveau, Jean-Paul, "Le lexique identificateur géographique", dans J.-Fr. Bonnot (éd.), Paroles régionales : normes, variétés linguistiques et contexte social, Strasbourg, 1995, p. 353-369.

Chauveau, Jean-Paul, "La disparition du subjonctif à Terre-Neuve, Saint-Pierre et Miquelon et en Bretagne: propagation ou récurrence?", dans P. Brasseur (éd.), Français d'Amérique. Variation, créolisation, normalisation, Centre d'Études Canadiennes, Université d'Avignon, 1998, p.105-119.

Chauveau, Jean-Paul, "Sur les emprunts lexicaux du québécois au percheron", à pa- raître dans les actes du cinquième colloque Français du Canada - Français de France, tenu en juin 1997 à Bellême (Perche) et organisé par Marie-Rose Simoni-Aurembou.

Chauveau, Jean-Paul / Lavoie, Thomas, "À propos des origines dialectales du lexique québécois", dans Revue de linguistique romane 57, 1993, p. 373-420.

Chauveau, Jean-Paul / Lavoie, Thomas, "Rapports lexicaux entre l'est québécois et l'ouest du domaine d'oïl", dans Thomas Lavoie (éd.), Français $d u$ Canada - Français de France, Actes $d u$ quatrième Colloque international de Chicoutimi, Québec, du 21 au 24 septembre 1994, Tübingen, Niemeyer, 1996, p. 47-60 (Canadiana romanica 12).

Dagenais, Louise, "Les sources historiques des diphtongues dans des dialectes d'oïl: de la parenté linguistique", dans Revue québécoise de linguistique théorique et appliquée 5, 1986, p. 63-128.

Dagenais, Louise, "L'émergence des diphtongues dans le français du Québec: hypothèse", dans Hans-Josef Niederehe et Lothar Wolf (éditeurs), Français $d u$ Canada - Français de France. Actes $d u$ troisième Colloque international d'Augsbourg du 13 au 17 mai 1991, Tübingen, Niemeyer, 1993, p. 3-16 (Canadiana romanica 7).

Faribault, Marthe, "L'emprunt aux langues amérindiennes", dans Hans-Josef Niederehe et Lothar Wolf (éditeurs), Français du Canada - Français de France. Actes du troisième Colloque international d'Augsbourg du 13 au 17 mai 1991, Tübingen, Niemeyer, 1993, p. 199-215 (Canadiana romanica 7).

Flikeid, Karin, La Variation phonétique dans le parler acadien du nord-est du Nouveau-Brunswick. Étude sociolinguisti- 
que, New York, Peter Lang, 1984 (American University Studies, Series XIII, Vol. 1).

Flikeid, Karin, "Les parlers acadiens de la Nouvelle-Écosse (Canada): diversification ou origines diverses?", dans Br. Horiot (éd.), Français du Canada Français de France. Actes $d u$ deuxième Colloque international de Cognac du 27 au 30 septembre 1988, Tübingen, Niemeyer, 1991, p. 195-214 (Canadiana romanica 6).

Flikeid, Karin, "Origines et évolution du français acadien à la lumière de la diversité contemporaine", dans Mougeon / Beniak 1994, p. 275-326.

Gauthier, Pierre / Lavoie, Thomas (dir.), Français de France et français $\mathrm{du} \mathrm{Ca}$ nada. Les parlers de l'Ouest de la France, du Québec et de l'Acadie, Université Lyon III Jean Moulin, Centre d'Études linguistiques Jacques Goudet, 1995 (Série dialectologie 3).

Gendron, Jean-Denis, "Effets linguistiques de l'intervention de l'Etat en matière de langue au Québec: la création d'un usage dominant", dans Hans-Josef Niederehe et Lothar Wolf (éditeurs), Français du Canada - Français de France. Actes du Colloque de Trèves du 26 au 28 septembre 1985, Tübingen, Niemeyer, 1987, p. 349-363 (Canadiana romanica 1 ).

Gendron, Jean-Denis, "La conscience linguistique des Franco-Québécois depuis la Révolution tranquille", dans Langue et identité. Le français et les francophones d'Amérique du Nord, textes et points de vue présentés par Noël Corbett, Québec, Les Presses de l’Université Laval, 1990.

Gérin, Pierre, "Norme lexicale et français acadien", dans Les marques lexicographiques en contexte québécois, Actes de la Table ronde tenue à Montréal les 3 et 4 novembre 1994, publiés par Louis Mercier et Claude Verreault avec la collaboration de Hélène CajoletLaganière et Geneviève Prévost, Gouvernement du Québec, 1998, p. 5158.

Heller, Monica, Crosswords. Language, Education and Ethnicity in French Ontario, Berlin / New York, Gruyter, 1994 (Contributions to the Sociology of Language 66).

Jaenen, Cornelius J. (dir.), Les Franco-Ontariens, Ottawa, Les Presses de l'Université d'Ottawa, 1993.

Juneau, Marcel, "Les plus anciens anglicismes lexicaux en franco-canadien", dans Bulletin des jeunes romanistes, Strasbourg, n 16, déc. 1969, p. 33-39.

Juneau, Marcel, "Reviviscence en ancien québécois de mots gallo-romans sous l'influence de l'anglais", dans Revue de linguistique romane 35,1971 , p. 388 392.

Juneau, Marcel, Contribution à l'histoire de la prononciation française au Québec. Étude des graphies des documents d'archives, Québec, Les Presses de l'Université Laval, 1972.

Kaltz, Barbara, "Le fransaskois: bilan historique et situation actuelle", dans $\mathrm{P}$. Brasseur (éd.), Français d'Amérique. Variation, créolisation, normalisation, Centre d'Études Canadiennes, Université d'Avignon, 1998, p. 131146.

Lacroix, Jean-Michel, "Réalités démographiques et politiques de l'immigration au Québec: l'état des lieux au début des années 1990", dans Études québécoises: bilan et perspectives. Actes $d u$ Colloque scientifique à l'occasion du quinzième anniversaire 
du "Centre d'Études Québécoises à l'Université de Trèves", 2-5 décembre 1993, Tübingen, Niemeyer, 1996, p. 165-178 (Canadiana Romanica 11).

Lang, Georges, "Le lexique du français des voyageurs dans le jargon tchinouk d'avant 1846", dans Revue québécoise de linguistique théorique et appliquée, 1995, p. 247-264.

Lapierre, André, "Parcours toponymiques de l'Amérique française", dans Poirier 1994, p. 227-236.

Magord, André, Une minorité francophone hors Québec: les Franco-Terreneuviens, Tübingen, Niemeyer, 1995 (Canadiana Romanica 10) [c.r. Chauveau RLiR 60, 1996, p. 602-604 et Bagola ZrP 114, 1998, p. 567-572].

Morin, Yves-Charles, "Les [ع] longs devant [s] en français: sources historiques et évolution", Revue québécoise de linguistique 20, 1991, p. 11-33.

Morin, Yves-Charles, "Les sources historiques de la prononciation du français du Québec", dans Mougeon / Beniak 1994, p. 199-236.

Morin, Yves-Charles, "The origin and development of the pronunciation of French in Québec", dans H. F. Nielse et L. Schøsler (éd.), Proceedings from the Second Rask Colloquium, Odense University, November 1994, Odense University Press, 1996, p. 243-275.

Mougeon, Raymond, "Le français en Ontario: bilinguisme, transfert à l'anglais et variabilité linguistique", dans Didier de Robillard et Michel Beniamino (éditeurs), Le français dans l'espace francophone, Paris, Champion, 1993, t. I, p. 53-77.

Mougeon, Raymond, "Recherche sur les origines de la variation vas, m'as, vais en français québécois", dans Thomas Lavoie (éd.), Français du Canada - Français de France, Actes du quatrième Colloque international de Chicoutimi, Québec, du 21 au 24 septembre 1994, Tübingen, Niemeyer, 1996, p. 61-77 (Canadiana romanica 12).

Mougeon, Raymond / Beniak, Édouard (publié par), Le français canadien parlé hors Québec. Aperçu sociolinguistique, Sainte-Foy (Québec), Les Presses de l'Université Laval, 1989 (collection Langue française au Québec, section 1, $\mathrm{n}^{\circ} 10$ ).

Mougeon, Raymond / Beniak, Édouard (publié par), Les origines du français québécois, Sainte-Foy (Québec), Les Presses de l'Université Laval, 1994.

Niederehe, Hans-Josef, "Quelques aspects de la morphologie du francoterreneuvien", dans Br. Horiot (éd.), Français du Canada - Français de France. Actes du deuxième Colloque international de Cognac du 27 au 30 septembre 1988, Tübingen, Niemeyer, 1991, p. 215-233 (Canadiana romanica $6)$.

Noël, Danièle, Les questions de langue au Québec (1759-1850), s.1., Éditeur officiel du Québec, 1990 (Dossiers du Conseil de la langue française 32).

Papen, Robert A., "Le parler français des Métis de l'Ouest canadien", dans P. Brasseur (éd.), Français d'Amérique. Variation, créolisation, normalisation, Centre d'Études Canadiennes, Université d'Avignon, 1998, p. 147-161.

Péloquin-Faré, L., "Les attitudes des FrancoAméricains de la Nouvelle-Angleterre envers la langue française", dans Corbett 1990, p. 145-155.

Péronnet, Louise, Le parler acadien du SudEst du Nouveau-Brunswick. Éléments 
grammaticaux et lexicaux, New York, Peter Lang, 1989 (American University Studies, Series IV, Vol. 8).

Péronnet, Louise, "La situation du français en Acadie: de la survivance à la lutte ouverte", dans Didier de Robillard et Michel Beniamino (éditeurs), Le français dans l'espace francophone, Paris, Champion, 1993, t. I, p. 101-116.

Péronnet, Louise, "Le français acadien", dans Gauthier / Lavoie 1995, p. 399-439.

Péronnet, Louise, "Nouvelles variétés de français parlé en Acadie du NouveauBrunswick", dans Les Acadiens et leur(s) langue(s): quand le français est minoritaire, Actes du colloque sous la direction de Lise Dubois et Annette Boudreau, Centre de recherche en linguistique appliquée, Université de Moncton, Les Éditions d'Acadie, 1996, p. 121-135.

Poirier, Claude, "L'anglicisme au Québec et l'héritage français", dans Travaux de linguistique québécoise, Sainte-Foy (Québec), Les Presses de l'Université Laval, 1978, t. 2, p. 43-106.

Poirier, Claude (dir.), Langue, espace, société. Les variétés du français en Amérique du Nord, avec la collaboration de Aurélien Boivin, Cécyle Trépanier et Claude Verreault, Sainte-Foy (Québec), Les Presses de l'Université Laval, 1994.

Poirier, Claude, "Les causes de la variation géolinguistique du français en Amérique du Nord. L'éclairage de l'approche comparative", dans Poirier 1994:69-95.

Poirier, Claude, "Le français au Québec", dans Histoire de la langue française, 1914-1945, sous la dir. de G. Antoine et R. Martin, Paris, CNRS-Éditions, 1995, p. 761-790.
Prévost, Geneviève, "Les chroniques de langage d'Étienne BLANCHARD (18831952). Aperçu des préoccupations normatives d'une époque au Canada", dans Cahiers de lexicologie 68, 1996, p. 175-192.

Roby, Yves, Les Franco-Américains de la Nouvelle-Angleterre (1776-1930), Sillery (Québec), Septentrion, 1990.

Rochet, Bernard, "Tendances phonétiques du français parlé en Alberta", dans Poirier 1994, p. 433-455.

Senécal, André, "Journalisme et création romanesque en Nouvelle-Angleterre francophone, 1875-1936", dans Poirier 1994, p. 145-154.

Verreault, Claude, "Inclusion, reconnaissance et identification des francismes dans les dictionnaires québécois : problèmes et méthodes à la lumière de l'expérience du Dictionnaire québécois d'aujourd'hui", dans Français du Canada - Français de France, Actes du quatrième Colloque international de Chicoutimi, Québec, du 21 au 24 septembre 1994, publiés par Thomas Lavoie, Tübingen, Niemeyer, 1996, p. 199-208 (Canadiana romanica 12 ).

Verreault, Claude / Lavoie, Thomas, "Genèse et formation du français au Canada: l'éclairage de la géographie linguistique", dans Revue de linguistique romane 60, 1996, p. 413-462.

Vézina, Robert, "Réciprocité de l'emprunt lexical en anglais américain et en français québécois", dans Langues et Linguistiques, Québec, Université Laval, 1993, p. 205-23.

Vézina, Robert, "Les gallicismes nord-américains en anglais des États-Unis: exploration d'un phénomène historique", à 
paraître dans Anglicisme et identité québécoise, Québec, Les Presses de l’Université Laval.

Wolf, Lothar, Französische Sprache in Kanada, Munich, Vögel, 1987.

\subsection{Documents, recueils et autres sources.}

Bouthillier, Guy / Meynaud, Jean, Le choc des langues au Québec. 1760-1970, Montréal, Les Presses de l'Université du Québec, 1972.

Corbett, Noël, Langue et identité. Le français et les francophones d'Amérique du Nord, textes et points de vue présentés par Noël Corbett, Québec, Les Presses de l'Université Laval, 1990.

Fedstats (U.S. Census Bureau): www. fedstats.gov

La situation de la langue française au Québec. Rapport de la commission d'enquête sur la situation de la langue française et sur les droits linguistiques au Québec, Québec, Gouvernement du Québec, 1972, 3 vol. (Livre I: La langue de travail. La situation $d u$ français dans les activités de travail et de consommation des Québécois. Livre II: Les droits linguistiques. Livre III: Les groupes ethniques. Les autres groupes ethniques et l'épanouissement du français au Québec.)

Statistique Canada: WWW.StatCan.CA/

André Thibault, Bâle 
Tableau 1a: Langue maternelle (Canada).

Anglais

16890615

$59,2 \%$

Français

6636660

$23,3 \%$

Autres langues

4598290

$16,1 \%$

Réponses multiples

402560

$1,4 \%$

Total

28528125

$100,0 \%$

Tableau 1b: Langue maternelle (par provinces et territoires).

\begin{tabular}{|c|c|c|c|c|}
\hline & T.-N. & Î.-P.É. & N.-É. & N.-B. \\
\hline Anglais & 538695 & 124805 & 836240 & 473260 \\
\hline Français & 2275 & 5550 & 35040 & 239730 \\
\hline Autres langues & 5465 & 2060 & 24495 & 10290 \\
\hline Réponses multiples & 725 & 440 & 4195 & 6345 \\
\hline \multirow[t]{2}{*}{ Total } & 547160 & 132855 & 899970 & 729625 \\
\hline & Québec & Ontario & Manitoba & Saskatchewan \\
\hline Anglais & 586435 & 7694635 & 813055 & 816955 \\
\hline Français & 5700150 & 479285 & 47665 & 19075 \\
\hline Autres langues & 657580 & 2296570 & 220855 & 126785 \\
\hline Réponses multiples & 100920 & 172300 & 18715 & 13800 \\
\hline \multirow[t]{2}{*}{ Total } & 7045085 & 10642790 & 1100290 & 976615 \\
\hline & Alberta & C.-B. & Yukon & T.-N.-O. \\
\hline Anglais & 2159275 & 2785020 & 26405 & 35835 \\
\hline Français & 52380 & 53040 & 1110 & 1355 \\
\hline Autres langues & 423810 & 801755 & 2700 & 25920 \\
\hline Réponses multiples & 33730 & 49940 & 435 & 1015 \\
\hline Total & 2669195 & 3689755 & 30650 & 64125 \\
\hline
\end{tabular}

Source: Statistique Canada, Recensement 1996. 
Tableau 2a: Langue d'usage à la maison (Canada).

Anglais

19031335

$66,7 \%$

Français

6359505

$22,3 \%$

Autres langues

2556830

$8,9 \%$

Réponses multiples

580460

$2,1 \%$

Total

28528125

$100,0 \%$

Tableau 2b: Langue d'usage à la maison (par provinces et territoires).

\begin{tabular}{|c|c|c|c|c|}
\hline & T.-N. & Î.-P.É. & N.-É. & N.-B. \\
\hline Anglais & 542270 & 128985 & 864235 & 498845 \\
\hline Français & 880 & 2910 & 19970 & 219385 \\
\hline Autres langues & 3270 & 545 & 11695 & 4070 \\
\hline Réponses multiples & 740 & 415 & 4070 & 7325 \\
\hline \multirow[t]{2}{*}{ Total } & 547160 & 132855 & 899970 & 729625 \\
\hline & Québec & Ontario & Manitoba & Saskatchewan \\
\hline Anglais & 710970 & 8773295 & 960120 & 917065 \\
\hline Français & 5770920 & 287190 & 22015 & 5380 \\
\hline Autres langues & 411010 & 1323850 & 95115 & 41390 \\
\hline Réponses multiples & 152185 & 258455 & 23040 & 12780 \\
\hline \multirow[t]{2}{*}{ Total } & 7045085 & 10642790 & 1100290 & 976615 \\
\hline & Alberta & C.-B. & Yukon & T.-N.-O. \\
\hline Anglais & 2410655 & 3152455 & 29070 & 43365 \\
\hline Français & 15725 & 14085 & 490 & 550 \\
\hline Autres langues & 198575 & 447855 & 755 & 18700 \\
\hline Réponses multiples & 44240 & 75360 & 335 & 1510 \\
\hline Total & 2669195 & 3689755 & 30650 & 64125 \\
\hline
\end{tabular}

Tableau 3: Le bilinguisme au Canada et dans chaque province et territoire.

\begin{tabular}{|c|c|c|c|c|c|}
\hline & Total & $\begin{array}{r}\text { Anglais } \\
\text { seulement }\end{array}$ & $\begin{array}{c}\text { Français } \\
\text { seulement }\end{array}$ & $\begin{array}{l}\text { Anglais } \\
\text { et français }\end{array}$ & $\begin{array}{l}\text { Ni l'un } \\
\text { ni l'autre }\end{array}$ \\
\hline Canada & $\begin{array}{r}28528125 \\
100,0 \%\end{array}$ & $\begin{array}{r}19134245 \\
67,1 \%\end{array}$ & $\begin{array}{r}4079085 \\
14,3 \%\end{array}$ & $\begin{array}{r}4841320 \\
17,0 \%\end{array}$ & $\begin{array}{r}473475 \\
1,6 \%\end{array}$ \\
\hline T.-N. & 547160 & 525190 & 155 & 21260 & 550 \\
\hline Î.-P.-É. & 132855 & 118075 & 170 & 14570 & 35 \\
\hline N.-É. & 899970 & 813320 & 1370 & 83980 & 1295 \\
\hline N.-B. & 729625 & 417970 & 73410 & 237765 & 475 \\
\hline Québ. & 7045085 & 358505 & 3951710 & 2660590 & 74275 \\
\hline Ont. & 10642790 & 9116165 & 46940 & 1234895 & 244790 \\
\hline Man. & 1100290 & 983820 & 1495 & 103140 & 11840 \\
\hline Sask. & 976615 & 920555 & 345 & 50770 & 4945 \\
\hline Alb. & 2669195 & 2455075 & 1615 & 178505 & 33995 \\
\hline C.-B. & 3689755 & 3342350 & 1780 & 248585 & 97045 \\
\hline Yukon & 30650 & 27340 & 50 & 3210 & 50 \\
\hline
\end{tabular}


T.-N.-O.

64125

55880

45

4035

4170

Source: Statistique Canada, Recensement 1996. 
Tableau 4: Le français (langue d'usage à la maison) en Nouvelle-Angleterre.

État

Connecticut

Maine

Massachusetts

New Hampshire

Rhode-Island

Vermont

Total
Valeur absolue

53586

81012

124973

51284

31669

17171

359695

* Sujets âgés de cinq ans et plus.

Source: Fedstats (U.S. Census Bureau, Census 1990).

$\begin{array}{rr}\text { Valeur relative } & \text { Population totale* } \\ 1,8 \% & 3060000 \\ 7,1 \% & 1142122 \\ 2,2 \% & 5605751 \\ 5,0 \% & 1024621 \\ 3,4 \% & 936423 \\ 3,3 \% & 521521 \\ & \\ 2,9 \% & 12290438\end{array}$

